\title{
Peptide-Enabled Targeted Delivery Systems for Therapeutic Applications
}

\author{
Mingpeng Liu'1,2,3, Xiaocui Fang ${ }^{1,3}$, Yanlian Yang ${ }^{1,3 *}$ and Chen Wang ${ }^{1,3 *}$ \\ ' CAS Key Laboratory of Biological Effects of Nanomaterials and Nanosafety, CAS Key Laboratory of Standardization \\ and Measurement for Nanotechnology, CAS Center for Excellence in Nanoscience, National Center for Nanoscience \\ and Technology, Beijing, China, ${ }^{2}$ Department of Chemistry, Tsinghua University, Beijing, China, ${ }^{3}$ University of Chinese \\ Academy of Sciences, Beijing, China
}

\section{OPEN ACCESS}

Edited by:

Xiaoguang Wang,

The Ohio State University,

United States

Reviewed by:

Weiqi Zhang,

Chinese Academy of Medical Sciences and Peking Union Medical

College, China

Mingzhan Wang,

University of Chicago, United States

*Correspondence:

Yanlian Yang

yangyl@nanoctr.cn

Chen Wang

wangch@nanoctr.cn

Specialty section:

This article was submitted to

Biomaterials,

a section of the journal

Frontiers in Bioengineering and

Biotechnology

Received: 28 April 2021

Accepted: 11 June 2021

Published: 01 July 2021

Citation:

Liu M, Fang $X$, Yang $Y$ and Wang C (2021) Peptide-Enabled

Targeted Delivery Systems

for Therapeutic Applications.

Front. Bioeng. Biotechnol. 9:701504.

doi: 10.3389/fbioe.2021.701504
Receptor-targeting peptides have been extensively pursued for improving binding specificity and effective accumulation of drugs at the site of interest, and have remained challenging for extensive research efforts relating to chemotherapy in cancer treatments. By chemically linking a ligand of interest to drug-loaded nanocarriers, active targeting systems could be constructed. Peptide-functionalized nanostructures have been extensively pursued for biomedical applications, including drug delivery, biological imaging, liquid biopsy, and targeted therapies, and widely recognized as candidates of novel therapeutics due to their high specificity, well biocompatibility, and easy availability. We will endeavor to review a variety of strategies that have been demonstrated for improving receptor-specificity of the drug-loaded nanoscale structures using peptide ligands targeting tumor-related receptors. The effort could illustrate that the synergism of nano-sized structures with receptor-targeting peptides could lead to enrichment of biofunctions of nanostructures.

Keywords: targeting peptide, nanostructure, enhanced receptor-specificity, drug delivery, tumor therapy

\section{INTRODUCTION}

Traditional small molecule drugs often suffer from various biopharmaceutical delivery obstacles such as non-specific distribution and inadequate accumulation at the site of interest. These limitations could be overcome by using appropriate strategies for directing drugs to specific disease tissues. In this field, nanotechnology has illustrated immense potentials in the past few decades, such as controlled drug release, promoted targeting drug delivery, and so on (Patra et al., 2018). Compared with bulk materials, nanostructures possess ultra-small sizes and large specific surface areas that can distinctively interact with biological interfaces. Their unique properties and benefits over bulk materials have been widely explored and exploited in the biomedical fields (McNamara and Tofail, 2017; Han et al., 2019).

Generally, the strategies based on nanostructures for effective drug delivery to the destination parts mainly include passive and active targeting. The passive targeting is mainly achieved by the enhanced permeability and retention (EPR) effect, which could be attributed to the abnormal structures of tumor vessels (Matsumura and Maeda, 1986). However, the therapeutic efficacies of cancer therapies based on passive targeting are far from optimal. One of the reasons is that the EPR effect is yet to be rigorously established and has limitations due to the EPR effect is 
highly diverse across both different tumor types and different subregions of a single tumor tissue (Bjornmalm et al., 2017).

The practicability of active targeting tumor therapy has been verified in many studies. By introducing receptorspecific ligands such as antibodies, aptamers, small molecules, and peptides, nanostructures with active targeting capability can be constructed. These active targeting delivery systems can direct payloads to the tumor parenchyma by specific recognition between tumor-associated antigens and targeting ligands. Among these receptor-specific ligands, antibodies and other large protein-based ligands possess the best binding affinity, but they usually suffer from several limitations including the immunogenicity, large size $(\sim 150 \mathrm{KDa})$ and non-specific clearance by the reticuloendothelial system (RES) and liver, resulting in poor passive diffusion across capillary endothelial cell membranes and dose-limiting systemic toxicity (Aina et al., 2002). Therefore, the successful application of macromolecular compounds, such as monoclonal antibodies, is limited to the vascular endothelium tumor (Thorpe, 2004) and hematological malignancies (Reff et al., 2002).

In comparison, bioactive peptides have a much smaller molecular weight $(<10 \mathrm{KDa})$. Despite only $1 \sim 10 \%$ of binding affinity compared with parent antibodies (Zhang et al., 2012), peptides provide superior advantages including lower immunogenicity, stronger penetration, lower production cost, and easier synthesis and modification. Additionally, the large specific surface area of nanostructures allows peptides to present multiple copies at the same time to obtain higher binding affinity, and also offers the possibility to incorporate different peptide ligands in a single construct. Compared with small molecule ligands, peptides have higher diversity, specificity, and targeting capability (Vlieghe et al., 2010; Sasikumar and Ramachandra, 2018; Ganesan et al., 2019). With these factors, peptides have been widely investigated as candidates for novel drugs.

At the same time, peptide drugs also suffer from unfavorable obstacles for clinical application including susceptibility to digestion by proteases and rapid plasma clearance (several minutes to several hours of half-life). To overcome these obstacles, various nanostructures have been utilized as carriers for peptide drug delivery, which could enhance solubility and circulation lifetime of peptides, and promote their specific accumulation into target tissues (Vo et al., 2012; Maeda et al., 2013; Bozzuto and Molinari, 2015). Such strategy could be achieved through encapsulating peptides into the core of nanostructures, or chemically conjugating peptides over the surface of nanostructures, thus obtaining peptide-enabled nanostructures with significant gain.

The synergism of peptides and nanostructures could strengthen their favorable characteristics of each technology and overcome natural limitations of individual materials. Many efforts for constructing various peptide-enabled nanostructures for targeted drug delivery have been reported over the past few decades. In this review, we mainly provide an overview of recent advances in the synergism of nanostructures with receptortargeting peptides that lead to enrichment of biofunctions of nanostructures and reduction of material limitations. It should be noted that due to the limited space, we will outline some representative peptide-enabled targeted delivery systems and related applications in cancer therapies. In order to have a comprehensive view, interested readers are encouraged to consult other related articles and reviews in this issue.

\section{THE CONSTRUCTION OF PEPTIDE-ENABLED NANOSTRUCTURES}

\section{Conjugation Strategies}

In general, biomolecule-nanoparticle conjugates will either be covalent or non-covalent in nature. The former includes covalent coupling of biomolecules to the surface or surface ligands of nanostructures, while the latter includes non-covalent encapsulation and self-assembled nanostructures driven by noncovalent interaction (Figure 1).

The covalent conjugations of peptides and other biomolecules to the surface of nanoparticles (NPs) are usually driven by the chelation of metal ions or Au-thiol dative bonds. The conjugation to surface ligands of NPs is usually accomplished through functional group coupling reactions. Common examples include the conjugation of maleimide with thiols, and the condensation of carboxyls with amines mediated by $N$-hydroxysuccinimidyl (NHS) along with carbodiimide (EDC). However, when there are multiple reaction groups on the ligands, it will lead to diverse patterns of the connection, which often affects the expected bioactivity. So, many researchers utilized orthogonal click reactions to construct multifunctional NPs. Functional groups that do not naturally exist in endogenous proteins, such as the azido group and alkenyl group, are introduced into the ligands. In this way, conjugation with higher selectivity, more controllable orientation, and fewer by-products can be obtained (Beal and Jones, 2012).

In general, cysteine-containing peptides can be linked to the surface of AuNPs directly. However, such binding may affect the bioactivity of peptides, therefore a sulfhydryl connecting piece is usually added between peptides and NPs. Commonly utilized linker includes cysteine, cystine, mercaptopropionic acid, and glutathione, etc. (Gao et al., 2012). Moreover, since dative bonds are reversible and weaker than typical covalent bonds, monothiol coatings drastically affected by ligand exchange during blood circulation (Kassam et al., 2006). Therefore, peptide coatings with multi-dentate binding sites of NPs have been increasingly explored. Such peptides possess multiple cysteine residues or bidentate dithiol ligands, providing multiple anchoring points to the surface of NPs (Krpetic et al., 2009; Xu et al., 2012). It could be anticipated that such strategy could lead to higher propensity for reattachment and reformation of the initial construct.

In most cases, non-covalent attachment between biomolecules and NPs refers to electrostatic interaction, hydrophobic interaction, hydrogen bond, and $\pi-\pi$ stacking forces. For instance, amphiphilic molecules, often lipids, can self-assemble into diverse nanostructures under specific conditions, depending on their natural properties, recognition, and interaction with solvent molecules. The stabilities of non-covalent bindings are dictated by equilibrium dissociation constants so that biomolecule-nanoparticle conjugates are quite sensitive to the 


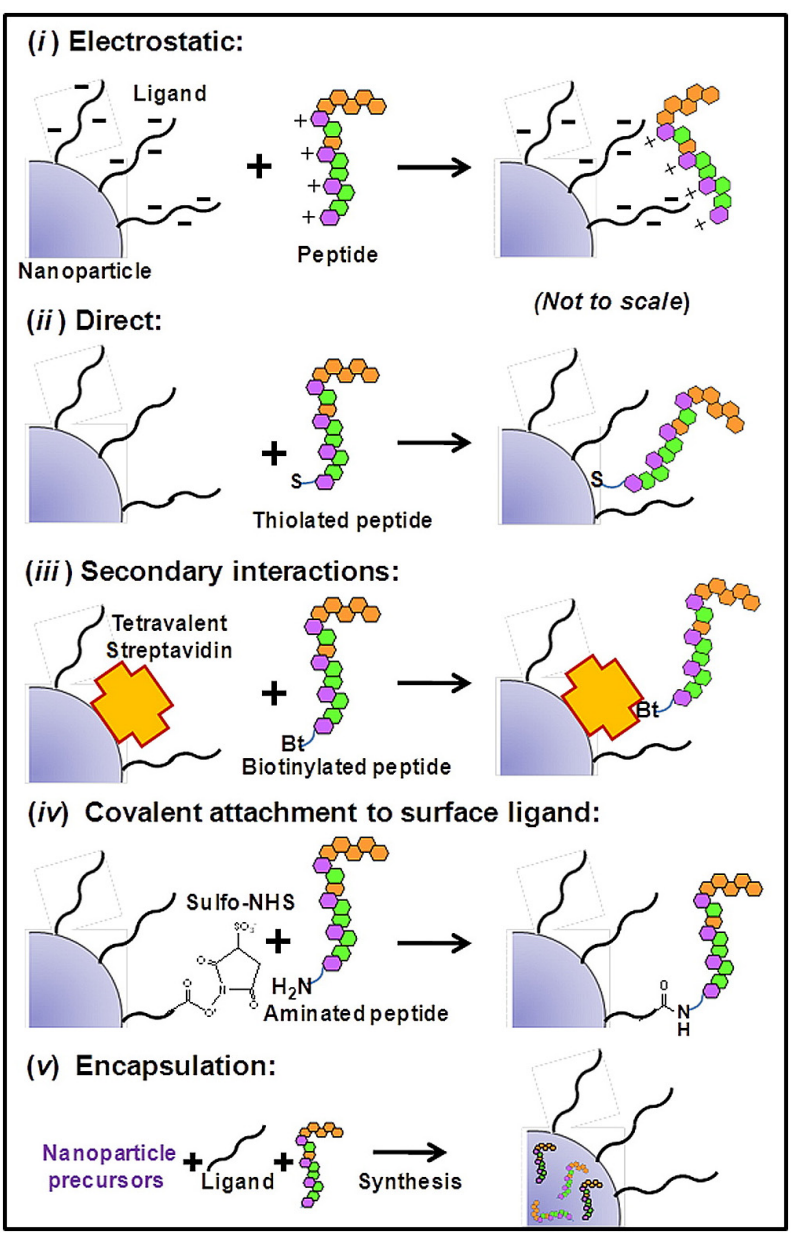

FIGURE 1 | Schematics of generally used bioconjugation attachment generally to NPs: (i) Electrostatic interaction. Peptides and NP surface with opposite charges can mediate charge-charge-based attachment; (ii) Direct interaction. Some amino acids (e.g., Cysteine) can bind to the surface of AuNPs with high binding affinity via Au-thiol dative bonds (iii) Secondary interactions. Specific non-covalent interactions such as biotin-streptavidin interactions can mediate direct binding of biotinylated peptides to SA-functionalized NPs (iv) Covalent chemical attachment. Classical conjugation chemistry include the conjugation of maleimide with thiols, condensation of carboxyls with amines mediated by NHS/EDC, and various click-chemistry reactions. (v) Encapsulation. Such self-assembled encapsulation may be driven by hydrophobic interaction (Sapsford et al., 2013). Copyright 2013 American Chemical Society.

concentrations of NPs and biomolecules during preparation and application. These approaches provide advantages such as controllable self-assembly preparation and drug release ( $\mathrm{Ma}$ et al., 2016). In addition to non-specific physical binding, there are some highly specific and stable non-covalent binding modes, such as the specific interaction between biotin and streptavidin. However, although avidin-biotin coupling has been widely used in the formation of an essentially irreversible and specific linkage between biological macromolecules, this conjugation strategy still has some drawbacks that need to be optimized, such as non-specific interactions for diagnostic assays.
Compared with non-covalent bonding, covalent bonding is more stable. When covalent conjugates are introduced into the complicated biological environments, the targeting ligands covalently coupled to nanoparticles are hard to be destroyed or covered up. However, as for non-covalent conjugates, targeting ligands are more likely to be replaced by various proteins in biological environments. On the other hand, such non-covalent conjugates provide advantages such as easy preparation, usually requiring only stoichiometric mixing of each component. Also, the property of revisable dissociation makes them suitable for designing controlled drug release systems.

\section{Interaction Between Biomolecules and Interface of Nanostructures}

The in vivo performance of NPs is closely related to many parameters. Understanding the interaction between nanostructures and biological systems is of fundamental significance. The chemical composition, distribution characteristics, and regulation mechanism on nano-biological interfaces provide the basis for the successful construction of expected biological nanostructures. In general, in addition to natural covalent or non-covalent interaction between biomolecules and nanostructures mentioned earlier, the size and shape of nanostructures, along with the ligand length and ligand density, contribute a lot to their interaction.

For example, many researches showed that the cover ratio of surface molecules will increase with the decrease of the size of NPs (Katari et al., 1994; Chen and Kimura, 1999). These observations may be due to the fact that when the particle size decreases, the radius of the particle begins to contribute to the minimum space required by a surface molecule (Terrill et al., 1995; Love et al., 2005). In addition, the shape of NPs also regulates the adsorption of surface molecules. Manna et al. (2003) demonstrated that organic surfactants would preferentially coat specific faces of a crystal and decreased the energy of these faces. By this effect, they successfully achieved controlled growth of Co nanodiscs and CdSe nanorods (Manna et al., 2003). Nakano et al. (2014) using nucleic acids as model ligands, and demonstrated that the density of ligands on a material surface is not the higher the better. A modest probe density would provide maximum hybridization rate and efficiency, which represents the best binding affinity (Nakano et al., 2014). Another interesting example is the melittin, a positive antimicrobial peptide of 26 amino acids. At low concentrations, the peptide is monomeric and irregularly curled. Once associated with lipid membranes, melittin rearranges by hydrophobic interactions and assumes an $\alpha$-helical conformation. By investigating the interaction of melittin with phospholipids in liposomes, lipid disks, and micelles, the results showed that melittin tended to adsorb on the highly curved edge of lipid disks (Lundquist et al., 2008).

When there is more than one kind of ligand, the situation becomes more complex. For instance, Kuna et al. (2009) demonstrated that mixed ligand molecules have unique assemble modes on the surface of AuNPs. The ligand mixture selfassembled into large homo-ligand domains, and further selfassembled into stripe-like domains on AuNPs. These stripe-like 
domains belong to a unique phase separation mode. Many other researches explored the influence factors of the self-assemble structures and phase separation structures of molecules on the surface of NPs, and indicated that the ligand length, ratio of different ligands, compatibility of ligand terminal groups, volume of terminal functional group, and curvature radius of the local surface could influence the phase separation structures (Jackson et al., 2004; Singh et al., 2007, 2011; Tung and Cacciuto, 2013; Yu et al., 2020; Wang et al., 2021). In general, the performance of ligand-decorated NPs is related to many parameters, not only the natural characteristics of NPs and targeting ligands, but also their proportion and connection modes.

\section{FUNCTIONAL ENHANCEMENT OF NANOPARTICLES ENABLED BY PEPTIDES}

\section{Peptide-Enhanced Biological Stability of Nanoparticles}

In order to reach the target site and achieve expected functions, NPs should circulate for enough time in the bloodstream, while avoiding premature removal by the RES and renal clearance. Albanese et al. showed that AuNPs become agglomerated when absorbed serum proteins under physiological conditions, leading to different cellular uptake patterns between single and aggregated nanoparticles (Albanese and Chan, 2011). Besides, the cores of some commonly used NPs are usually heavy metallic and should be capsuled carefully to prevent the leaching of harmful metal ions. Therefore, peptide coatings that could cover metal cores to enhance stability and reduce toxicity are important components of NPs for approaching practical clinical application (Libralato et al., 2017).

The peptide modification onto the surface of NPs has been widely employed to provide biological stabilization to NPs. Common strategies mainly include adjusting the surface charge and hydrophobicity, using biological anti-phagocytic signaling to enhance retention, or promoting the formation of a favorable protein corona such as a pre-formatted albumin corona to prevent the attachment of alternative proteins (Spicer et al., 2018). For example, reduced glutathione has been widely used for the synthesis of AuNPs with good monodispersity and stability (Wu et al., 2014). The functionalization of the peptide of CALNN could endow AuNPs with stability by negatively charged peptide corona to withstand aggregation (Levy et al., 2004). Multidentate peptides such as the hexa-histidine motif ( $\mathrm{His}_{6}$ ) can form stable metal coordinates with AuNPs and quantum dots (QDs), providing a stable coverage with excellent resistance to desorption (Prasuhn et al., 2010; Aldeek et al., 2013).

\section{Peptide-Promoted Cell Penetration and Targeting of Nanoparticles}

To accumulate into the site of interest, NPs should overcome a series of biological barriers. They need to escape the clearance of the body's immune system, bypass vascular endothelia, accumulate into the target tissue, and finally recognize and enter into the target cells. Since these biological barriers help the biological system to maintain controlled and ordered material transport, the target tissue and cell penetration of foreign NPs remains one of the major challenges for therapeutic applications (Blanco et al., 2015). In recent years, many cell penetrating peptides (CPPs) with the ability to promote receptor-mediated endocytosis and the following endosomal escape have been widely elaborated (Xie et al., 2020). It is estimated that over 800 of CPPs have been studied (Gautam et al., 2012). The most commonly used CPPs are highly cationic and bind to negatively charged cell membrane through the electrostatic interaction, and then mediate internalization via either passive diffusion or endocytic pathway. Typical examples of CPPs include the TAT (Schwarze et al., 1999), $\mathrm{R}_{8}$ (Yang et al., 2018), penetratin (Jiang et al., 2019), and pHLIP peptide (Thevenin et al., 2009).

For instance, the TAT (GRKKRRQRRRPQ) sequence is originally derived from the human immunodeficiency virus and has been demonstrated to deliver drug-loaded nanocarriers including liposomes, polymeric micelles, and metal NPs into cells. Interestingly, the D-enantiomer of TAT and disordered TAT sequence also show the ability to mediate cell penetration (Futaki et al., 2001; Qin et al., 2012), suggesting that the positive charges of CPPs play a vital role in enhancing cell internalization, rather than specific receptor-induced internalization (Futaki et al., 2001; Qin et al., 2012). Importantly, although cationic peptides enhance cell uptake efficiently, endosomal escape may be hindered by the strong electrostatic attraction, which may partly account for the fact that the efficiency of cargos to deliver to the cytosol remains relatively low (Verdurmen et al., 2017). To enhance endosomal escape efficiency, Morshed et al. (2016) attached TAT peptide to AuNPs via an acid-labile hydrazine bond that would cleave spontaneously after reaching acidic endosomal environments, which greatly promoting the endosome escape of AuNPs. In general, for designing CPPs-functionalized NPs, the balance of cell penetration and endosomal escape ability should be considered carefully.

\section{Peptide-Promoted Tumor Penetration and Targeting of Nanoparticles}

Numerous peptides named tumor homing peptides have been widely identified. They have the potential to penetrate tumor tissues, resulting in an extensive distribution across the tumor mass. For example, Teesalu et al. reported a class of peptides (named as CendR) that share an $(\mathrm{R} / \mathrm{K}) \mathrm{XX}(\mathrm{R} / \mathrm{K})$ motif in the $\mathrm{C}$-terminus and specifically target the neuropilin-1 receptor, a cell membrane receptor that regulates vascular permeability and angiogenesis, as well as the development of the nervous system (Teesalu et al., 2009). Interestingly, this motif must be exposed at the C-terminus of the peptides to remain the biological activity. The CendR sequence can be embedded in a long peptide sequence and activated by an appropriate protease cleavage.

Up to date, the RGD sequence and its cyclic derivative (cRGD) might be the most widely used tumor homing sequences, which direct to integrin $\alpha_{\mathrm{v}} \beta_{3}$, a signaling protein that is overexpressed in many types of endothelial tumors (Desgrosellier and Cheresh, 2010). To show the synergy between the two peptides, 
Sugahara et al. (2009) constructed a cyclic fusion sequence of $\mathrm{c}(\mathrm{CRGDK} / \mathrm{RGPD} / \mathrm{EC})$ that triggers tissue penetration of drugs through three processes: The RGD motif targets integrin $\alpha v \beta 3$ to accumulate at tumor endothelium cells, then exposes the cryptic CendR motif, RGDK/R, by proteolytic cleavage, and finally induces tumor penetration following interaction with neuropilin1. In recent years, RGD modified NPs have been exploited rapidly. Xie et al. developed cRGD-decorated semiconducting polymer nanoparticles for photoacoustic imaging (Xie et al., 2017). The results showed that with the targeting capability of cRGD, NPs could effectively delineate the tumor sites in living mice and increased the photoacoustic intensity. Fan et al. also demonstrated that RGD modification could enhance the specific targeting ability of self-assembled fluorescent nanoparticles and improve their anti-tumor activity (Fan et al., 2018).

In addition to tumor homing peptides, a variety of peptides that has high binding affinities to target receptors overexpressed on specific cells have been widely used to realize cell targeting. The GE11 peptide (YHWYGYTPQNVI) is a typical example, which is firstly identified by Li et al. (2005) through the screening of phage display libraries. The GE11 peptide has been demonstrated to promote tumor penetration and selectively bind to the epidermal growth factor receptor (EGFR), a typical overexpressed receptor in epithelial origin tumors. A series of subsequent researches confirmed that GE11 could act as an excellent allosteric EGFR ligand without mitogen activity. GE11 peptide-mediated drug delivery systems including liposomes, polymeric micelle, and viruses have been developed for disease diagnosis and drug delivery. A summary of GE11 peptideenabled targeted delivery systems has been elaborately discussed in the review of Genta et al. (2018).

In most cases, selected ligands will promote the specific accumulation at targeting sites. For example, encouraged by our previous work that peptide E5 (GGRSFFLLRRIQGCRFRNTVDD) was first identified as an antagonist to chemokine receptor CXCR4 with effective antimetastasis activity ( $\mathrm{Li}$ et al., 2014). In a subsequent study, Duan et al. (2016) demonstrated that E5 can also bind to human serum albumin (HSA) with a high affinity and presented enhanced interaction between HSA and CXCR4-overexpressed tumor cells mediated by E5. Compared with free HSA, the $K_{\mathrm{D}}$ value of E5-HSA nanocomplex to CXCR4-positive cells improved by approximately an order of magnitude, and showed improved cell migration inhibitory effect. Their results indicate that a single peptide sequence can be multi-functional. On one hand, E5 can serve as a targeting ligand to endow nanostructures specific binding affinity to CXCR4. Indeed, $\mathrm{Zu}$ et al. (2020) subsequently demonstrated that E5 modified QDs also showed much higher binding affinity to Hela cells compared with free E5 (The equilibrium dissociation, $K_{\mathrm{D}}: 15.5 \mu \mathrm{M}$ and $125 \mathrm{nM}$, respectively). On the other hand, HSA is the most abundant protein in the plasma, and their binding will not affect the normal function of E5. These properties indicate the formation of a favorable protein corona for E5-decorated NPs, preventing the attachment of other alternative proteins. That may partly decrease the unexpected influence of the rebellious protein corona and prolonging the circulation time.
Moreover, since E5 is originally a therapeutic peptide with anti-metastasis activity, their synergism provides an excellent platform for affinity-controlled drug release systems. On the whole, this study has shown that compared with passive targeting NPs, the peptide ligand-directed NPs present improved therapeutic performances in various degrees. However, it is worth noting that the development of ligand directed NPs remains in its infancy. At present, there was no liganddirected active targeting nanomedicine has been approved for clinical use.

\section{Peptide-Enabled Controlled Drug Release of Nanoparticles}

Peptide-drug conjugates (PDCs) are an emerging strategy for delivering payloads to target tissues while decreasing unexpected effects to healthy tissues. Drugs are covalently attached to specific peptides via cleavable linkers to make prodrugs. This could temporarily mask or limit the drugs' bioactivity and minimize premature drug liberation. The diversity of peptide sequence can not only enable the facile preparation of various kinds of PDCs with unique specificity, but also regulate the hydrophobicity and ionization of the whole conjugates, which contribute greatly to the bioavailability. At present, there are two PDC drugs (Lutathera and Melflufen) that have been approved by Food and Drug Administration (FDA) and many candidates are being evaluated in various stages of clinical development (Cooper et al., 2021). In this field, advanced design is so-called one-component nanomedicine, which means amphiphilic peptide-drug conjugates self-assembling to form their own nanostructure as drug delivery vehicles (Ma et al., 2016). Active drugs can release in control over time or initiate by some specific stimulus after introducing into biological environments.

For example, Yang et al. (2019) fabricated a self-assembling active targeting anticancer hydrogel by conjugating both chlorambucil and tyroservatide to a self-assembling peptide sequence. They demonstrated that a heating-cooling process would easily urge the compound monomer to self-assemble into a nanofiber structured hydrogel. The hydrogel could concurrently deliver the two drugs with controlled release and prolonged plasma circulation half-life, leading to enhanced cell uptake and antitumor activity.

Peptides can also be utilized for engineering affinity-controlled release systems, which could achieve controlled diffusion of drugs through the different binding affinities between molecules (Pakulska et al., 2016). In general, elaborated drug-binding ligands, such as peptides, oligonucleotides, or proteins, are immobilized onto a polymer matrix. Then therapeutic drugs can bind to these ligands through preferred non-covalent interactions. An equilibrium is established between free drugs and ligand-bound drugs. By adjusting the concentration of the ligand and the binding strength of the ligand with the drug, the rate of drug release can be regulated to meet different requirements. In this field, a series of potential affinity peptides have been identified by in vitro selection and directed evolution. Now a database of affinity peptides (MimoDB) 
(Huang et al., 2012) has been established, which may contribute to affinity-controlled release applications.

\section{PEPTIDE-ENABLED NANOSTRUCTURES OF TARGETED DELIVERY SYSTEMS}

\section{Peptide-Mediated Liposomal Delivery Systems}

Liposomes are closed spherical vesicles with a lipid bilayer structure, which is similar to the cell membrane. This unique structure allows effective encapsulation of both hydrophilic and hydrophobic molecules. Such encapsulation improves the stability of loaded drugs and reduces their systemic toxicity. Liposomes provide substantial advantages over many other nanocarriers, including consistent size, efficient drug loading, excellent steric stabilization, and biocompatibility (Noble et al., 2014). In the past few decades, liposome-based nanomedicines have been considerably explored. Several liposomal drugs have achieved efficacious clinical outcomes (Crommelin et al., 2020). For instance, the liposomal doxorubicin $\left(\right.$ Doxil $\left.^{\circledR}\right)$ is the first FDAapproved liposomal therapy for cancer treatment, providing improved survival rate and life quality for patients over free doxorubicin (Barenholz, 2012).

The liposomal delivery system can be facilitated by targeting peptides to mediate specific drug delivery, leading to increased drug penetrability and specific accumulation at the site of interest. Such sequence can be easily functionalized to the surface of liposomes via reactive lipid head groups, to obtain peptideenabled targeted drug-loaded liposomes. After extravasating into the tumor interstitial space via the EPR effect, liposomes can be internalized into specific target cells by ligand-receptor recognition. These peptide-functionalized liposomes combine both passive and active delivery mechanisms, which could exhibit better drug delivery efficiency than drug-containing liposomes without peptide modification (Wilhelm et al., 2016). Until now, a variety of engineered liposomes by the introduction of targeting peptides have been successfully designed to deliver cargoes to specific organs, tissues, and tumor cells through multiple targeting (Jiang et al., 2012; Roy et al., 2017; Huang et al., 2018; Lu et al., 2019).

For instance, in order to design a hepatotropic liposome, Witzigmann et al. (2019) selected and optimized a derivative peptide of Myrcludex B that derived from the hepatitis B virus large envelope protein. Myrcludex B-derived peptides could specifically target the sodium-taurocholate cotransporting polypeptide (NTCP/SLC10A1) that overexpressed on the hepatocyte sinusoidal membrane (Yan et al., 2012; Witzigmann et al., 2019). On this basis, they further designed five Myrcludex B-derived peptides with different sequences and acyl chain modifications, and modified these peptides onto the surface of liposomes (Figure 2A). The shorter fatty acid chain (CappreS2-48, C10) seems to lead to more differentiated and unstable liposomes (Figure 2B) than the longer fatty acid chain (MyrpreS2-48, C14). This phenomenon may be attributed to the shorter acyl chain interferes with the liposome stability by backward bending insertion into liposomal membranes. Some earlier researches also have shown that shorter lipid anchors were less stable in liposomal membranes, promoting faster dissociation and re-association with neighboring lipids (Webb et al., 1998; Sauer et al., 2006). As shown in Figure 2C, the cellular uptake of Myr-preS2-48A modified-liposomes is not related to the level of NTCP expression, suggesting that NPLGFFP plays an important role in NTCP-specific targeting. In addition, the longer fatty acid chain modification onto the surface of liposomes at the end of the peptide represents the higher binding affinity to NTCP-overexpressing HepG2 cells. The peptide of preS2-48 without fatty acid modification did not possess a considerable binding affinity to NTCP receptors.

Subsequently, the peptide ligand density of liposomes can be regulated by the added amounts of coupled maleimide group of $P_{2 E}{ }_{2000}$-DSPE chain. The results showed that the cellular uptake of peptide modified liposomes is ligand concentrationdependent, and at least $0.25 \mathrm{~mol} \%$ of ligand concentration (MyrpreS2-31) is needed for efficient active targeting (Figure 2D). When Myr-preS2-31- and Myr-preS2-48-modified liposomes are introduced into the transgenic zebrafish embryos at identical ligand densities, liposomes modified with shorter peptides (MyrpreS2-31) show better systemic circulation compared with longer ligand modified liposomes (Figure 2E), which may because the longer sequence indicates higher immune clearance. Similarly, the decreased systemic circulation time and higher clearance rate also counteract the advantage of higher ligand densities (0.5 mol\%) (Figure 2F). Under the comprehensive balance between the target capability and biocompatibility, a $0.25 \mathrm{~mol} \%$ of Myr-preS2-31 ligand modification would exhibit the best performance (Figure 2G).

On the whole, various elements can influence the interaction between nanomaterials and bio-interface. That may partly account for the fact that all clinically approved liposome-based nanomedicines are non-ligand directed, which totally relies on passive targeting to achieve tumor accumulation. Although many positive results have been reported in animal models, few positive effects in patients were published for such ligandtargeted liposomes (Crommelin et al., 2020). It is laborious but important to carefully evaluate these effects and search for the best balance.

Similarly, Zou et al. (2018) developed a vemurafenib-loaded liposome for the precise inhibition of subcutaneous melanoma via the skin. The TD peptide (ACSSSPSKHCG) endowed liposomes with the ability to target subcutaneous melanoma cells harboring BRAF mutation. It can temporarily open the paracellular pathway and promote the drug to penetrate the skin (Chen et al., 2006). Compared with oral administration or intravenous injection, transdermal delivery performed a better biological effect in enhancing antitumor efficacy and reducing damage to normal organs.

Zhao et al. (2020) described a double-modified doxorubicinencapsulating liposome (AAN-FnBPA5-Dox). The AAN motif was covalently bind to the N-terminal of the FnBPA5 peptide. Then, such tandem peptide was covalently linked to the doxorubicin-encapsulated liposomes via amide bonds. The FnBPA5 peptide has a high binding affinity to relaxed Fn 
A
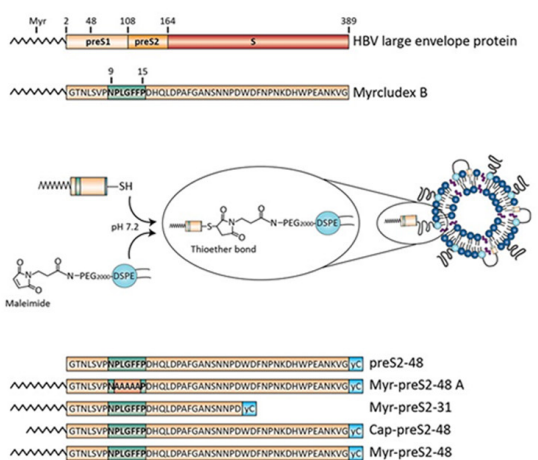

C

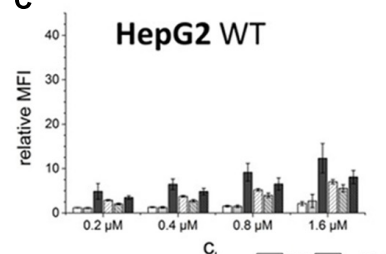

E
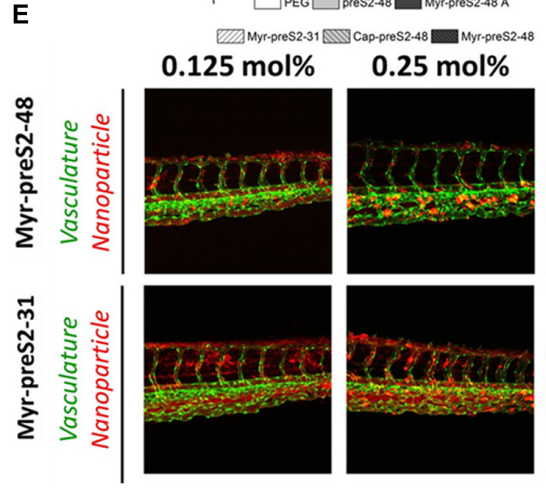

B
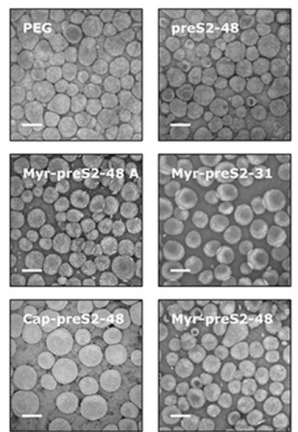

$\mathbf{F}$
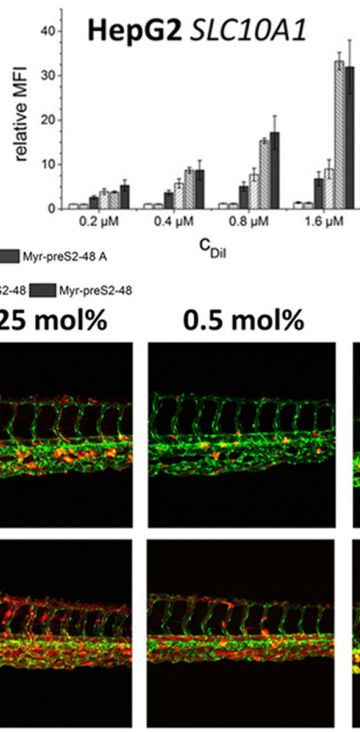

$1.0 \mathrm{~mol} \%$

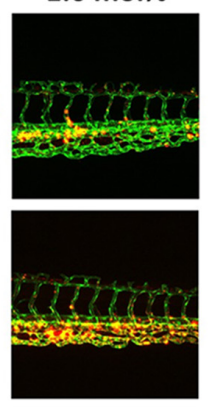

D
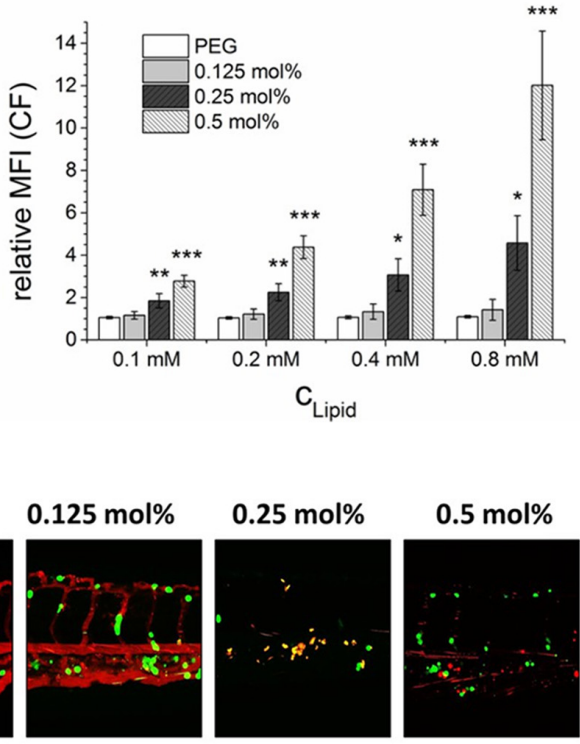

$0.5 \mathrm{~mol} \%$
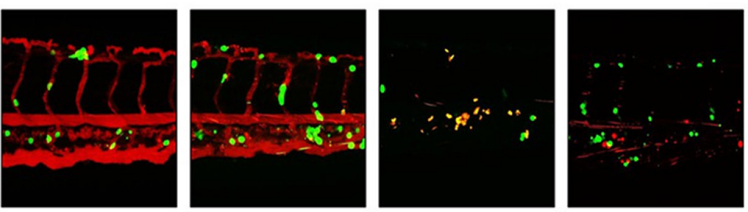

G

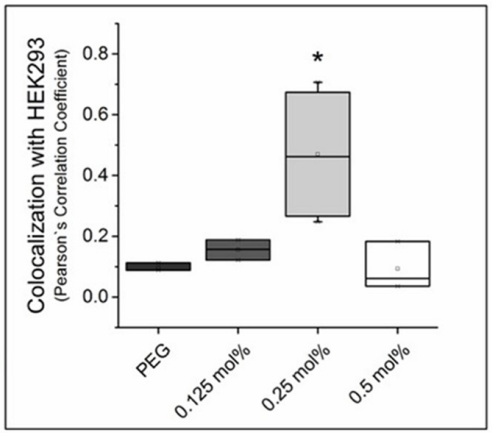

FIGURE 2 | Identifying different peptide ligands for a better NTCP-specific targeting. (A) Schematic representation of HBV large envelope protein, Myrcludex B and its five derivatives. Peptides were conjugated to the end of PEG chain integrated in the liposomes through the connection of sulfhydryl group with maleimide group. (B) The morphological characters of different Myrcludex B-derived lipopeptide conjugated liposomes. (C) The uptake rate of different peptide conjugated liposomes into HepG2 wild type cells and SLC10A1 overexpressing HepG2 cells was identified by flow cytometry analysis. (D) The uptake of liposomes modified with different amounts of Myr-preS2-31 identified by flow cytometry analysis. Significance $\left({ }^{\star} p<0.05,{ }^{* *} p<0.01\right.$, and $\left.{ }^{* \star *} p<0.001\right)$ was calculated relative to PEG, respectively. (E) The performance of Myr-preS2-48- and Myr-preS2-31-modified liposomes was tested in zebrafish embryos expressing green fluorescent protein in their vasculature endothelial cells (green signal). Dil (red signal) was used to label the membrane of liposomes. (F) Myr-preS2-31-modified liposomes were tested in wild type zebrafish embryos xenotransplanted with human, GFP expressing HEK293 cells (green signal), expressing SLC10A1. Dil (red signal) was used to label the membrane of liposomes. Yellow signals demonstrate the colocalization of liposomes with HEK293-GFP cells. (G) Pearson's Correlation Coefficient (PCC) for quantitative analysis of liposomes binding to HEK293-GFP cells (Witzigmann et al., 2019). Significance $\left({ }^{*} p<0.05\right)$ was calculated relative to PEG. Open Access.

and collagen I in the extracellular environment and $\alpha$-SMAexpressing cancer-associated fibroblasts, reducing the density of collagen fibers around the tumor tissue, and promoting the cell penetration of doxorubicin. And the AAN motif was attached to extend the circulation time by hindering parts of the bioactivity of FnBPA5 sequence and reducing the accumulation in offtarget Fn-excreting organs. The results indicated that AANFnBPA5-Dox remained stable during internal circulation until the AAN motif was recognized and cleaved by legumain that overexpressed in tumor-associated macrophages (Song et al., 2016). In general, such modification would partly hinder the natural bioactivity of the cryptic sequence until the cover motif was cleaved. An earlier study claimed that the addition of the AAN moiety to TAT sequences would lead to a decrease transport efficacy by $72.65 \%$ until legumain catalysis recovered the penetrating capacity (Liu et al., 2014). In this way, they successfully formed an ecological therapy, which could extend the circulation time, regulate the tumor microenvironment, thus improve the therapeutic response.

\section{Peptide-Mediated Polymeric Micelle Delivery Systems}

Polymer micelles usually refer to self-assembled spherical colloidal nanoparticles formed by amphiphilic block copolymers. They usually possess a core-shell structure in an analogous fashion to liposomes in which poorly soluble cargos can be encapsulated. In terms of drug delivery, micellar-based 
systems are suitable for carrying hydrophobic molecules. The advantages of these structures include a simple preparation process, efficient drug loading, well biocompatibility, and controlled release kinetics (Long et al., 2015). The physical properties and biological activity of micelles can be regulated by the functionalization of flexible pendant groups. This makes it possible for synthesizing stimuli-responsive polymeric micelles that could be controlled to release cargos in response to various surroundings (Aura-Ileana et al., 2012). Thus, polymeric micelles are finding increasing utilization across many biomedical disciplines. However, it should be noted that a practical issue is that polymeric micelles tend to dissociate and release cargos when they are diluted and interact with biomolecules in the blood, often leading to premature cargo release (Bae and Yin, 2008).

For instance, Zheng et al. (2021) developed a peptide-directed micelle delivery system targeting tumor-associated antigen of CD36. Co-assembled DSPE-PEG 2000 micelles were modified with targeting peptide pep2 (RRGTIAFDNWVDTGTRVYD) and thus obtained appreciably enhanced specificity and sensitivity toward CD36-positive tumor cells. Compared with free pep2, the binding affinity of pep2-modified micelles to the CD36 receptor was enhanced by $\sim 30 \%$ (Figures 3A,B). Relatively, as shown in Figure 3C, compared with unmodified micelles (M-Dox), the intracellular delivery amount of doxorubicin was enhanced $\sim 3$ fold with the modification of pep2 (Pep2-M-Dox).

Another similar study utilized a D-peptide ligand for better proteolytic stability and demonstrated that the modification of micelles with D-peptide ligand could facilitate micelles to pass through the simulated blood-brain barrier and thus achieve deeper drug penetration (Mao et al., 2017). As shown in Figures 3D,E, compared with micelles without peptide modification, FALGEA modification showed higher recognition ability to EGFR-positive tumor cells, while the reverse sequence of FALGEA did not have such specificity. Moreover, compared with L-type FALGEA, the D-type FALGEA-modified micelles exhibited enhanced stability and targeting ability both in vitro and in vivo.

In addition to surface modification to promote specific tumor recognition and accumulation, peptides can also be encapsulated within micelles as a switch for drug directed release. Barve et al. developed a biodegradable, enzyme-responsive micelle with a block copolymer consisted of PEG, a matrix metalloproteinase-2 (MMP-2)-targeting peptide (PLGVRK), and cholesterol (Figure 3F; Barve et al., 2020). When the system was introduced into MMP-2 overexpressed tumor microenvironment of prostate cancer, the linker between cholesterol and PEG chain would be cleaved, which induces micelle dissociation and subsequent drug release of cabazitaxel. With this design, a controlled drug delivery system responding to the tumor microenvironment was constructed successfully.

Another interesting research showed the potential of antigen peptide mediated polymeric micelles as cancer nanovaccines to activate the body's active anti-tumor immunity (Liu et al., 2017). Liu et al. designed a type of micelle vaccine incorporating both two kinds of antigen peptides (E7 and OVA) and monophosphoryl lipid A (MPLA) as an immune adjuvant. The E7 (GQAEPDRAHYNIVTFCCKCD) and OVA peptide (SGLEQLESIINFEKL) were selected as tumor-associated antigens to activate specific tumor immune response (Liu et al., 2017). The results demonstrated that PEG-PE micelles could assist non- $\alpha$-helical structures of E7 and OVA refolding into $\alpha$ helix structures and enhance their cytosolic delivery, which could exhibit an impressive therapeutic antitumor effect (Fang et al., 2016). Their efforts provide a new design for cancer treatment, which is expected to significantly improve the application and benefit rate of cancer patients.

\section{Peptide-Guided Exosomal Delivery Systems}

Exosomes are a class of nanosized extracellular vehicles (EVs) secreted by living cells, showing great potentials in advanced drug delivery and disease treatment. Fundamentally, EVs are a universal form of signal transduction between cells. They circulate in the blood and transport various bioactive molecules to regulate cell function and behaviors in distant target cells (Kalluri and LeBleu, 2020). Therefore, compared with other artificial NPs, exosomes have a natural ability to deliver therapeutic molecules with advantages including minimal immune clearance and well biological barrier penetration (Barile and Vassalli, 2017). For example, Haney et al. (2015) exploited the ability of exosomes to cross the blood-brain barrier and developed a non-invasive approach to deliver catalase to the brain. They utilized intranasal administered exosomes loaded with catalase to cross the blood-brain barrier, and subsequently obtained promoted neuroprotective effects (Haney et al., 2015). So far, several clinical trials have been carried out to test the safety of exosome-based nanomedicine and some of them have shown satisfying outcomes in dendritic cell-derived exosomes (Pitt et al., 2016).

In recent years, peptide ligand enrichment on engineered exosomes has been investigated to target specific cell types. Compared with other artificial NPs, exosomes have their unique ligand modification strategy. The modification may be achieved by engineering the parent cells, leading to the natural exhibition of ligands on the surface of exosomes. For peptide ligands, their coding sequences can be inserted into a lentiviral expression plasmid and transfect into producer cells, and fused with an EV transmembrane protein, such as Lamp2b or PDGFR TM domain. Then the peptide will naturally display on the outer surface of exosomes produced by these cells (Dang and Zeng, 2016).

It has been generally proposed that exosomes are suitable for delivering nucleic acids and exchange genetic information between cells, containing both mRNA and microRNA (Valadi et al., 2007). Many researches show that exosomal mRNAs are functional and can regulate the phenotype of target cells (Valadi et al., 2007; Mittelbrunn et al., 2011; Kojima et al., 2018). Thus, engineered exosomes become ideal carriers for target genetic therapy. For example, Lydia et al. (AlvarezErviti et al., 2011) explored an endogenous brain-targeting exosome for siRNA delivery (Figure 4A). They cloned the sequence that encodes an acetylcholine receptor targeting peptide (YTIWMPENPRPGTPCDIFTNSRGKRASNG, RVG) into the N 
A

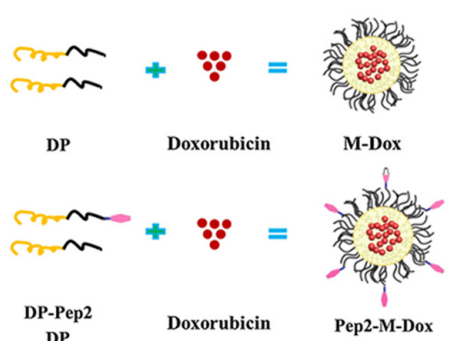

B

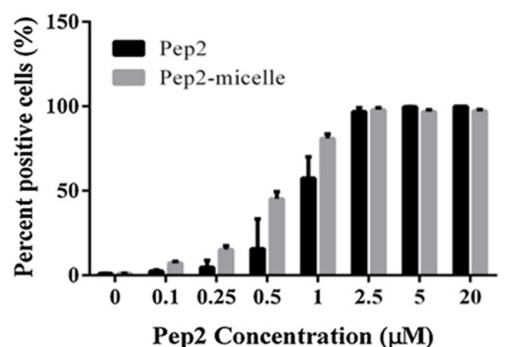

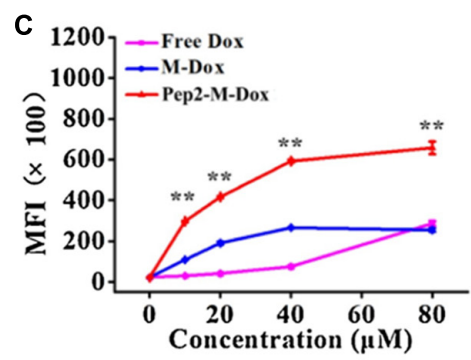
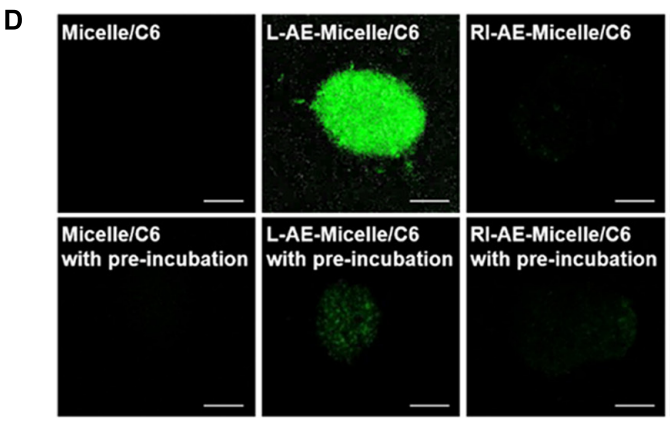

D-AE-Micelle/C6

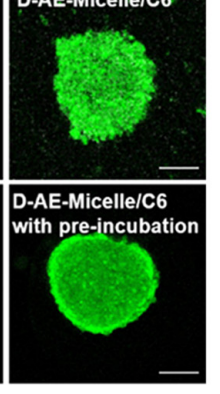

$\mathbf{F}$
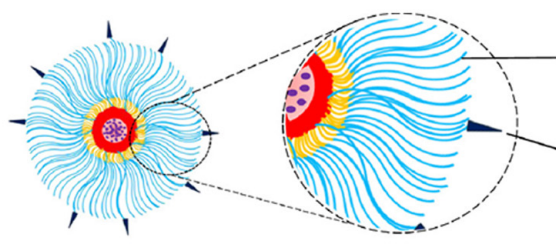

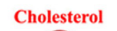

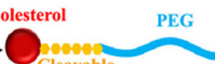

Cleavable
linker

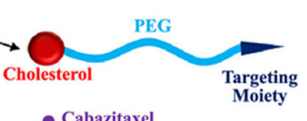

E

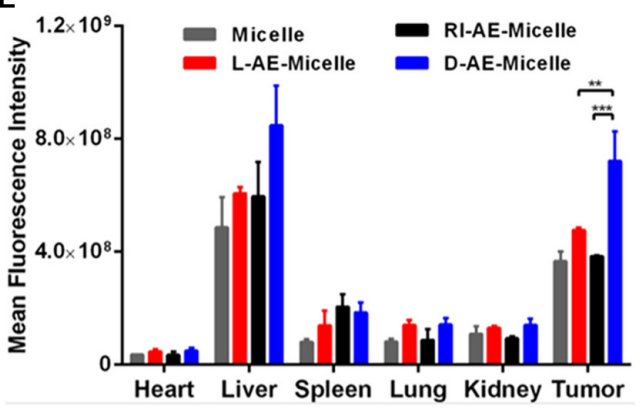

FIGURE 3 | Peptide-mediated polymeric micelles for therapeutic applications. (A) Schematics of the preparation of pep2 modified DSPE-PEG 2000 micelles. (B) The binding affinities of micelles with or without pep2 modification to HepG2 tumor cells, quantified by flow cytometry. (C) MFls of HepG2 cells treated by Free doxorubicin, Micelle-doxorubicin, or pep2-Micelle-doxorubicin at equivalent concentrations of doxorubicin (Zheng et al., 2021). Significance $\left({ }^{\star *} p<0.01\right)$ was calculated relative to M-Dox. Copyright 2021 Elsevier. (D) Transcytosis of modified micelles in BBB/U87 model in vitro. Penetration of different micelles in the BBTB/U87 tumor spheroid coculture model with or without preincubation were examined by a confocal microscope. L-AE: L-(FALGEA); D-AE: D-(FALGEA); RI-AE: D-(AEGLAF). (E) The fluorescence intensity of different micelles in tumor and organs were identified by semiquantitative ROI analysis (Mao et al., 2017). Copyright 2017 American Chemical Society. ${ }^{* *}$ and ${ }^{* \star *}$ indicate $p<0.01$ and $p<0.001$, respectively. (F) Schematic illustration of the MMP-2-responsive polymeric micelle (Barve et al., 2020). Copyright 2020 Elsevier.

terminus of murine Lamp2b protein on the exosomal membranes (Figure 4B), and then introduced the engineered plasmid into dendritic cells to express targeting exosomes. The results showed that these engineered exosomes possessed similar gene knockdown efficiency compared to transfection reagents with significant specificity (Figure 4C). As for the RVG peptidemodified exosomes, they exhibited decreased off-target effects, and great therapeutic potential by the strong knockdown efficacy in both mRNA and protein level (Figures 4D,E).

However, it should be noted that this peptide fusion is difficult to predict and control. Because of the fusion of targeting peptides into membrane proteins to form large compounds, poor expression, adverse folding, and incorrect display of these fusion proteins remain of potential concern. Furthermore, compared with other simpler NPs, exosomes are difficult to prepare, usually isolated from exosome-secreting cell lines by ultracentrifugation, which limits their large-scale application. In addition, the producer cell type, physiological state, and manufacturing protocols will vary the composition and properties of exosomes, making it difficult to characterize exosomes pharmaceutically (Barile and Vassalli, 2017). These characteristics remain the major obstacles in translating naturally secreted exosomes into clinical practice.

\section{Peptide-Modified Gold Nanoparticles (AuNPs)}

Gold nanostructures show outstanding potential with multifunctionality, such as antimicrobial, anticancer, drug delivery, sensing, and imaging. They are relatively inert and stable in biological environments, offering attractive tools for biomedical diagnostic and therapy (Dreaden et al., 2012; Ramalingam, 2019). Particularly, AuNPs exhibit unique localized surface plasmon resonance-related optical phenomena, which show strong light emission excited by concerted electron oscillation. The unique optical and electronic properties of AuNPs make them valuable tools for in vitro diagnostics, including nearinfrared fluorescence, enhanced magnetic resonance imaging, surface-enhanced Raman scattering, as well as photothermal and photoacoustic imaging (Boisselier and Astruc, 2009). Wu et al. (2019) summarized a brief review of recent advances of AuNPs in bioimaging, and herein, we mainly focus on the therapeutic applications of AuNPs. 


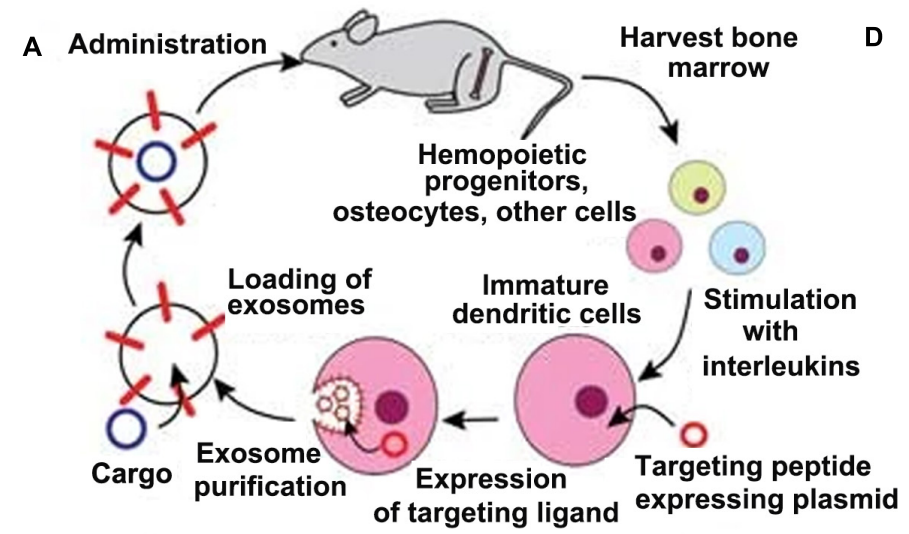

B

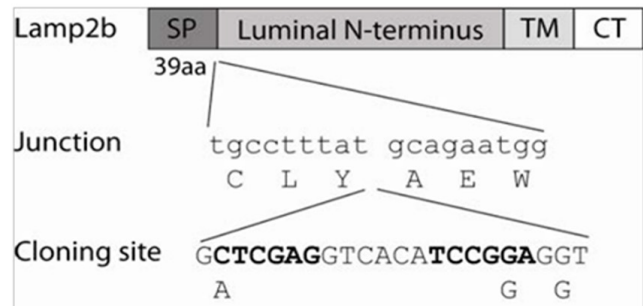

C

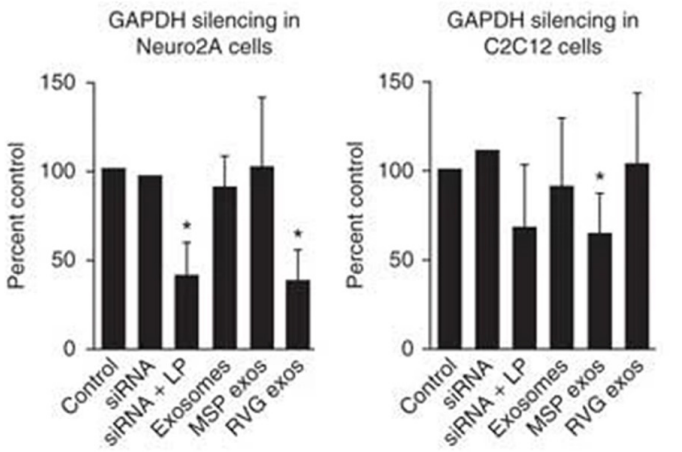

D
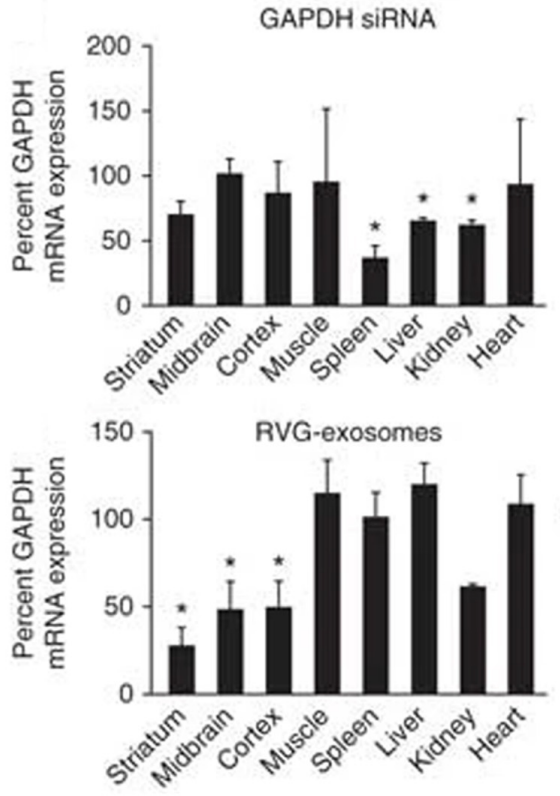

E
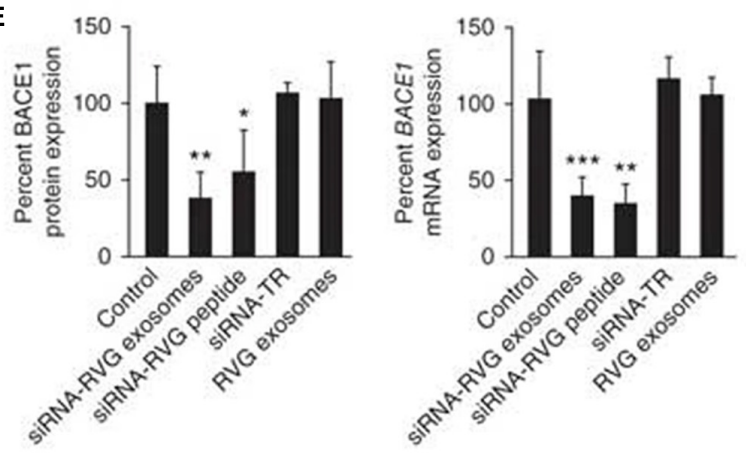

FIGURE 4 | Delivery of siRNA to the mice brain by peptide-mediated exosomes. (A) Schematic representation of the preparation of self-exosomes for gene delivery. (B) The modified Lamp2b protein. SP, signal peptide; TM, transmembrane domain; CT, C terminus. (C) qPCR of GAPDH in neuronal cells (Neuro2A) and murine muscle cells (C2C12) of medium, naked siRNA, siRNA delivered with Lipofectamine 2000, unmodified exosomes, MSP exosomes, and RVG exosomes. Significance $\left({ }^{*} p<0.05\right)$ was calculated relative to Control group, respectively. (D) The GAPDH qPCR in different organs of mice treated by naked GAPDH siRNA or encapsulated siRNA in RVG exosomes, normalized to untreated controls (100\%). Significance $\left({ }^{*} p<0.05\right)$ was calculated relative to untreated controls, respectively. (E) The BACE1 qPCR of mice brain cortical sections of five groups of free RVG exosomes, RVG exosomes encapsulated with BACE1 siRNAs, transfection reagent with BACE1 siRNA, and RVG-9R peptide with BACE1 siRNA, and compared with untreated control. All qPCR was normalized to 18S RNA levels. Significance $\left({ }^{*} p<0.05,{ }^{* \star} p<0.01\right.$, and ${ }^{* \star *} p<0.001$ ) was calculated relative to Control group, respectively (Alvarez-Erviti et al., 2011). Copyright 2011 Springer Nature.

Gold nanoparticles possess a unique photothermal effect. They can absorb the light near the NIR region and convert it to heat effectively, causing the death of malignant tumors with the advantage of minimal invasiveness (Beik et al., 2019). Noteworthy, endogenous biomolecules appear the minimal absorb in the NIR diagnostic windows $(650 \sim 900 \mathrm{~nm}$, and 1000 1350 nm) (Kobayashi et al., 2010). This allows photothermal therapy based on AuNPs to penetrate the body more deeply. Indeed, photothermal therapy is a central application of AuNPs and has been employed widely to destroy tumor cells. It is noteworthy that such localized destruction of pathological parts strongly relies on specific and adequate accumulation at the target site. Therefore, an effective targeting ligand such as peptide appears more of significance. Some recent examples referring to peptide-modified AuNPs in photothermal therapy are listed in the Table 1, some of them also loaded with other drugs for combination therapy.

Peptide-enabled AuNPs have also been used to direct payloads to their targets. Pal et al. reported a kind of AuNPs for plectin1 targeted gemcitabine delivery in pancreatic cancer (Pal et al., 2017). Plectin-1 is aberrantly overexpressed on the surface of pancreatic ductal adenocarcinoma while showing cytoplasmic expression in healthy cells. They used a multifunctional peptide sequence (KTLLPTPYC) as the targeting ligand, as well as the reducing agent. Such sequence was derived from a plectin-1 targeting sequence (KTLLPTP). A tyrosine residue is attached 
TABLE 1 | A brief summary of recent AuNPs in therapeutic applications.

\begin{tabular}{|c|c|c|c|c|}
\hline Peptide & Target & Loading & Delivery to & References \\
\hline KTLLPTPYC & Plectin-1 & Gemcitabine & Tumor cell & Pal et al., 2017 \\
\hline TAT & - & Cas9-sgRNA & Tumor cell & Wang et al., 2018 \\
\hline GE11 & EGFR & Pc 4 & Tumor cell & Meyers et al., 2015 \\
\hline RGD & Integrin $\alpha \vee \beta 3$ & - & Tumor blood vessels & Ali et al., 2017 \\
\hline $\begin{array}{l}\text { RGD and } \\
\text { CGGGPKKKRKVGG }\end{array}$ & Integrin $\alpha \vee \beta 3$ & - & The nucleus of tumor cell & Aioub and El-Sayed, 2016 \\
\hline CRQAGFSL & $\begin{array}{l}\text { Urokinase-type } \\
\text { plasminogen activator } \\
\text { receptor }\end{array}$ & 5-Aminolevulinic acid & pancreatic tumor cell & Li et al., 2017 \\
\hline $\begin{array}{l}\text { CPNFDWDPNNSNAGF } \\
\text { APDLQHDPFFGLP }\end{array}$ & $\begin{array}{l}\text { Squamous Cell Carcinoma } \\
\text { Antigen } 1\end{array}$ & - & $\begin{array}{l}\text { Hepatocellular Carcinoma } \\
\text { Cells }\end{array}$ & Jha et al., 2017 \\
\hline TSFAEYWALLSP & MDM2 and MDMX & - & $\begin{array}{l}\text { Tumor cells bearing } \\
\text { wild-type p53 }\end{array}$ & He et al., 2020 \\
\hline
\end{tabular}

at the C-terminal for reducing property while the cysteine residue is attached for coupling with AuNPs. This modified peptide plays the role of glutathione in traditional synthesis, to fabricate AuNPs via an in situ reduction, simplifying the synthesis process effectively. These AuNPs were further functionalized with gemcitabine through electrostatic interaction of the pyrimidine ring or reversible Au-N bond, which accounted for an initial burst drug release followed by a sustained release. In subsequent in vivo experiments, the sequence KTLLPTP promoted the spacer selectively accumulation in tumor tissues and thus leading to higher specific cytotoxicity to pancreatic ductal adenocarcinoma cells than chemotherapeutic drugs alone.

\section{CHALLENGES IN PEPTIDE-PROMOTED DELIVERY SYSTEMS FOR TUMOR THERAPIES}

At present, almost all clinically approved drug delivery systems are non-ligand directed, which totally relies on passive targeting to achieve tumor accumulation. Although many positive results have been reported in animal models, few positive effects on efficacy in patients were published for ligand-targeted NPs (Crommelin et al., 2020). Key challenges of clinical transformation include inadequate standard methods for clinical production and comprehensive characterization, an overall understanding of NPs function and behavior in the body, and the difficulties in the recapitulation of natural tumors in laboratory models (Belfiore et al., 2018). Some of them are waiting for technique development and some rely on foundational research. Herein, we focus on the potential of peptides in overcome such difficulties and highlight that a proper peptide coating may influence and regulate the formation and composition of a serum protein corona on the surface of NPs, which may contribute to a better performance in vivo.

The biophysical properties of NPs-based delivery systems after intravenous injection are an important consideration, including ligand stability, ligand function, circulation time, and clearance properties. After the introduction of NPs into the biological environment, the nanoparticle surface will rapidly interact with a mixture of biomolecules especially proteins to form a layer of the protein corona, which is dynamic and highly dependent on the biological environment (Monopoli et al., 2012). High abundant proteins form an initial corona and are gradually removed by tightly bound proteins to form thermodynamically favored hard corona. The performance of the protein corona is difficult to predict and often leads to unexpected influences of the fate of NPs in vivo with undesired activity or function (Corbo et al., 2017). It has been generally proposed that the presence of a protein corona around the surface of peptide-enabled targeted delivery systems may inhibit the binding affinity of targeting ligand with its receptor, reducing the targeting ability of the NPs, accounting for unanticipated biodistribution, pharmacokinetics, and efficacy in vivo (Walkey and Chan, 2012; Salvati et al., 2013).

In this field, a proper coating may modulate and control the formation and property of the protein corona. In general, opsonins such as immunoglobulin G, complement factor and fibrinogen, can be adsorbed on the surface of NPs and trigger macrophage recognition and phagocytosis elimination (Owens and Peppas, 2006; Gao and He, 2014). If this process is inhibited, the recognition and clearance of NPs by the immune system can be reduced. On the contrary, when dysopsonins such as serum albumins and lipoproteins are enriched on the surface of NPs, the recognition is blocked and the circulation time can be increased (Peng et al., 2013). In the past few years, stealth polymers such as polyethylene glycol (PEG) have been widely used for limiting opsonins adsorption and decreasing NPs clearance. Such stealth polymers can form a protective layer around the NPs and provide a steric barrier to adsorption (Moore et al., 2015). Some of them can also regulate the surface charge and hydrophobicity for better biocompatibility. In addition, peptides with high binding affinity to dysopsonins may promote the formation of a beneficial protein corona, which could decrease the rapid clearance of NPs. Chen et al. (2009) demonstrated an ultra-low fouling peptides with alternating positive $(\mathrm{K})$ and negative charge (E) residues. Such mixed charged sequence possesses strong hydration ability and leads to a hydrated layer on the surface of NPs, preventing non-specific protein adsorption. However, there is a contradiction between the stealth performance and targeting performance. Many researches show that a stealth 
coating is associated with reduced specific cellular uptake (Yu et al., 2012). On the whole, it is important to consider the delicate balance between biocompatibility and targeting capability that determines NPs fate when designing a modification coating. More peptide sequences with expected characteristics are waiting for design, selecting and identification.

\section{CONCLUSION}

Nanotechnologies have developed rapidly, and targeting peptides have also found increasing favor in this context. The synergism combines favorable characteristics of nano-sized structures with bioactive peptides, which could enrich the biofunction, and overcome the natural limitations of individual materials. Over the past few decades, nanoparticles conjugated with peptides have emerged as powerful tools for biomedical applications. Numerous peptide-functionalized nanomaterials have been developed for biomedicine applications, including drug delivery, biological imaging, liquid biopsy, and targeted therapies. Nanotechnology has already revolutionized the mode that we discover and administer new biomedicine. However, obstacles such as inadequate knowledge about nanostructure interacting with biological interfaces still remain profound.

\section{REFERENCES}

Aina, O. H., Sroka, T. C., Chen, M. L., and Lam, K. S. (2002). Therapeutic cancer targeting peptides. Biopolymers 66, 184-199. doi: 10.1002/bip.10257

Aioub, M., and El-Sayed, M. A. (2016). A real-time surface enhanced raman spectroscopy study of plasmonic photothermal cell death using targeted gold nanoparticles. J. Am. Chem. Soc. 138, 1258-1264. doi: 10.1021/jacs.5b10997

Albanese, A., and Chan, W. C. W. (2011). Effect of gold nanoparticle aggregation on cell uptake and toxicity. ACS Nano 5, 5478-5489. doi: 10.1021/nn2007496

Aldeek, F., Safi, M., Zhan, N., Palui, G., and Mattoussi, H. (2013). Understanding the self-assembly of proteins onto gold nanoparticles and quantum dots driven by metal-histidine coordination. ACS Nano 7, 10197-10210. doi: 10.1021/ nn404479h

Ali, M. R. K., Wu, Y., Tang, Y., Xiao, H. P., Chen, K. C., Han, T. G., et al. (2017). Targeting cancer cell integrins using gold nanorods in photothermal therapy inhibits migration through affecting cytoskeletal proteins. Proc. Natl. Acad. Sci. U.S.A. 114, E5655-E5663. doi: 10.1073/pnas.1703151114

Alvarez-Erviti, L., Seow, Y. Q., Yin, H. F., Betts, C., Lakhal, S., and Wood, M. J. A. (2011). Delivery of siRNA to the mouse brain by systemic injection of targeted exosomes. Nat. Biotechnol. 29, 341-U179. doi: 10.1038/nbt.1807

Aura-Ileana, M. V., Teresa, G.-Q., Rosa-Elvira, N. E. A., Laura-Susana, A. T., and Víctor, C. O. (2012). Polymeric and ceramic nanoparticles in biomedical applications. J. Nanotechnol. 2012, 1-10. doi: 10.1155/2012/936041

Bae, Y. H., and Yin, H. Q. (2008). Stability issues of polymeric micelles. J. Contr. Release 131, 2-4. doi: 10.1016/j.jconrel.2008.06.015

Barenholz, Y. C. (2012). Doxil - the first FDA-approved nano-drug: lessons learned. J. Contr. Release 160, 117-134. doi: 10.1016/j.jconrel.2012.03.020

Barile, L., and Vassalli, G. (2017). Exosomes: therapy delivery tools and biomarkers of diseases. Pharmacol. Ther. 174, 63-78. doi: 10.1016/j.pharmthera.2017.02.020

Barve, A., Jain, A., Liu, H., Zhao, Z., and Cheng, K. (2020). Enzyme-responsive polymeric micelles of cabazitaxel for prostate cancer targeted therapy. Acta Biomater. 113, 501-511. doi: 10.1016/j.actbio.2020.06.019

Beal, D. M., and Jones, L. H. (2012). Molecular scaffolds using multiple orthogonal conjugations: applications in chemical biology and drug discovery. Angew. Chem. Int. Ed. 51, 6320-6326. doi: 10.1002/anie.201200002
Cautious design and further exploration to improve the safety and efficacy of these nano-formulations would be a necessary and long-term project.

\section{AUTHOR CONTRIBUTIONS}

ML: conceptualization, data curation, and writing-original draft. XF: validation, writing-review and editing, and funding acquisition. YY and CW: conceptualization, resources, writingreview and editing, supervision, and funding acquisition. All authors contributed to the manuscript and approved the submitted version.

\section{FUNDING}

This work was supported by the Strategic Priority Research Program of Chinese Academy of Sciences (XDB36000000), National Natural Science Foundation of China (Nos. 51861135103, 21721002, 21773042, and 21790394), and the Key Research Program of Frontier Sciences, Chinese Academy of Sciences (QYZDJ-SSW-SLH048).

Beik, J., Khateri, M., Khosravi, Z., Kamrava, S. K., Kooranifar, S., Ghaznavi, H., et al. (2019). Gold nanoparticles in combinatorial cancer therapy strategies. Coord. Chem. Rev. 387, 299-324. doi: 10.1016/j.ccr.2019.02.025

Belfiore, L., Saunders, D. N., Ranson, M., Thurecht, K. J., Storm, G., and Vine, K. L. (2018). Towards clinical translation of ligand-functionalized liposomes in targeted cancer therapy: challenges and opportunities. J. Contr. Release 277, 1-13. doi: 10.1016/j.jconrel.2018.02.040

Bjornmalm, M., Thurecht, K. J., Michael, M., Scott, A. M., and Caruso, F. (2017). Bridging bio-nano science and cancer nanomedicine. ACS Nano 11, 9594-9613. doi: 10.1021/acsnano.7b04855

Blanco, E., Shen, H., and Ferrari, M. (2015). Principles of nanoparticle design for overcoming biological barriers to drug delivery. Nat. Biotechnol. 33, 941-951. doi: $10.1038 /$ nbt.3330

Boisselier, E., and Astruc, D. (2009). Gold nanoparticles in nanomedicine: preparations, imaging, diagnostics, therapies and toxicity. Chem. Soc. Rev. 38, 1759-1782. doi: 10.1039/b806051g

Bozzuto, G., and Molinari, A. (2015). Liposomes as nanomedical devices. Int. J. Nanomed. 10, 975-999. doi: 10.2147/ijn.s68861

Chen, S. F., Cao, Z. Q., and Jiang, S. Y. (2009). Ultra-low fouling peptide surfaces derived from natural amino acids. Biomaterials 30, 5892-5896. doi: 10.1016/j. biomaterials.2009.07.001

Chen, S. H., and Kimura, K. (1999). Synthesis and characterization of carboxylatemodified gold nanoparticle powders dispersible in water. Langmuir 15, 10751082. doi: 10.1021/la9812828

Chen, Y., Shen, Y., Guo, X., Zhang, C., Yang, W., Ma, M., et al. (2006). Transdermal protein delivery by a coadministered peptide identified via phage display. Nat. Biotechnol. 24, 455-460. doi: 10.1038/nbt1193

Cooper, B. M., Iegre, J., O’ Donovan, D. H., Halvarsson, M. O., and Spring, D. R. (2021). Peptides as a platform for targeted therapeutics for cancer: peptide-drug conjugates (PDCs). Chem. Soc. Rev. 50, 1480-1494. doi: 10.1039/d0cs00556h

Corbo, C., Molinaro, R., Tabatabaei, M., Farokhzad, O. C., and Mahmoudi, M. (2017). Personalized protein corona on nanoparticles and its clinical implications. Biomater. Sci. 5, 378-387. doi: 10.1039/c6bm00921b

Crommelin, D. J. A., van Hoogevest, P., and Storm, G. (2020). The role of liposomes in clinical nanomedicine development. What now? Now what? J. Contr. Release 318, 256-263. doi: 10.1016/j.jconrel.2019.12.023 
Dang, X. T., and Zeng, X. R. (2016). Targeted therapeutic delivery using engineered exosomes and its applications in cardiovascular diseases. Gene 575, 377-384. doi: 10.1016/j.gene.2015.08.067

Desgrosellier, J. S., and Cheresh, D. A. (2010). Integrins in cancer: biological implications and therapeutic opportunities. Nat. Rev. Cancer 10, 9-22. doi: $10.1038 / \mathrm{nrc} 2748$

Dreaden, E. C., Alkilany, A. M., Huang, X. H., Murphy, C. J., and El-Sayed, M. A. (2012). The golden age: gold nanoparticles for biomedicine. Chem. Soc. Rev. 41, 2740-2779. doi: 10.1039/c1cs15237h

Duan, H. Y., Zhu, L., Hou, J. F., Peng, J. X., Xie, H. Y., Lin, Y. C., et al. (2016). Dualaffinity peptide mediated inter-protein recognition. Organ. Biomol. Chem. 14, 11342-11346. doi: 10.1039/c6ob02292h

Fan, Z., Chang, Y., Cui, C., Sun, L., Wang, D. H., Pan, Z., et al. (2018). Near infrared fluorescent peptide nanoparticles for enhancing esophageal cancer therapeutic efficacy. Nat. Commun. 9:2605. doi: 10.1038/s41467-018-04763-y

Fang, X., Yang, T., Wang, L., Yu, J., Wei, X., Zhou, Y., et al. (2016). Nano-cagemediated refolding of insulin by PEG-PE micelle. Biomaterials 77, 139-148. doi: 10.1016/j.biomaterials.2015.11.007

Futaki, S., Suzuki, T., Ohashi, W., Yagami, T., Tanaka, S., Ueda, K., et al. (2001). Arginine-rich peptides - an abundant source of membrane-permeable peptides having potential as carriers for intracellular protein delivery. J. Biol. Chem. 276, 5836-5840. doi: 10.1074/jbc.M007540200

Ganesan, A., Ahmed, M., Okoye, I., Arutyunova, E., Babu, D., Turnbull, W. L., et al. (2019). Comprehensive in vitro characterization of PD-L1 small molecule inhibitors. Sci. Rep. 9:12392. doi: 10.1038/s41598-019-48826-6

Gao, H., and He, Q. (2014). The interaction of nanoparticles with plasma proteins and the consequent influence on nanoparticles behavior. Exp. Opin. Drug Deliv. 11, 409-420. doi: 10.1517/17425247.2014.877442

Gao, J., Huang, X. Y., Liu, H., Zan, F., and Ren, J. C. (2012). Colloidal stability of gold nanoparticles modified with thiol compounds: bioconjugation and application in cancer cell imaging. Langmuir 28, 4464-4471. doi: 10.1021/ la204289k

Gautam, A., Singh, H., Tyagi, A., Chaudhary, K., Kumar, R., Kapoor, P., et al. (2012). CPPsite: a curated database of cell penetrating peptides. Database J. Biol. Databases Curation. 2012:bas015. doi: 10.1093/database/bas015

Genta, I., Chiesa, E., Colzani, B., Modena, T., Conti, B., and Dorati, R. (2018). GE11 peptide as an active targeting agent in antitumor therapy: a minireview. Pharmaceutics 10:2. doi: 10.3390/pharmaceutics10010002

Han, X. J., Xu, K., Taratula, O., and Farsad, K. (2019). Applications of nanoparticles in biomedical imaging. Nanoscale 11, 799-819. doi: 10.1039/c8nr07769j

Haney, M. J., Klyachko, N. L., Zhaoa, Y. L., Gupta, R., Plotnikova, E. G., He, Z. J., et al. (2015). Exosomes as drug delivery vehicles for Parkinson's disease therapy. J. Contr. Release 207, 18-30. doi: 10.1016/j.jconrel.2015.03.033

He, W., Yan, J., Li, Y., Yan, S., Wang, S., Hou, P., et al. (2020). Resurrecting a p53 peptide activator - an enabling nanoengineering strategy for peptide therapeutics. J. Contr. Release 325, 293-303. doi: 10.1016/j.jconrel.2020.06.041

Huang, J., Ru, B., Zhu, P., Nie, F., Yang, J., Wang, X., et al. (2012). MimoDB 2.0: a mimotope database and beyond. Nucleic Acids Res. 40, D271-D277. doi: 10.1093/nar/gkr922

Huang, S.-L., Klegerman, M. E., Peng, T., Moody, M., Holland, C., Haworth, K., et al. (2018). Targeted ultrasound responsive liposomes for therapeutic delivery of anti-inflammatory drugs into the atherosclerotic arterial wall. Circulation $138,1178-1190$.

Jackson, A. M., Myerson, J. W., and Stellacci, F. (2004). Spontaneous assembly of subnanometre-ordered domains in the ligand shell of monolayer-protected nanoparticles. Nat. Mater. 3, 330-336. doi: 10.1038/nmat1116

Jha, S., Ramadori, F., Quarta, S., Biasiolo, A., Fabris, E., Baldan, P., et al. (2017). Binding and uptake into human hepatocellular carcinoma cells of peptidefunctionalized gold nanoparticles. Bioconjug. Chem. 28, 222-229. doi: 10.1021/ acs.bioconjchem.6b00441

Jiang, K., Hu, Y., Gao, X., Zhan, C. Y., Zhang, Y. Y., Yao, S. Y., et al. (2019). Octopus-like flexible vector for noninvasive intraocular delivery of short interfering nucleic acids. Nano Lett. 19, 6410-6417. doi: 10.1021/acs.nanolett. $9 \mathrm{~b} 02596$

Jiang, T. Y., Zhang, Z. H., Zhang, Y. L., Lv, H. X., Zhou, J. P., Li, C. C., et al. (2012). Dual-functional liposomes based on $\mathrm{pH}$-responsive cell-penetrating peptide and hyaluronic acid for tumor-targeted anticancer drug delivery. Biomaterials 33, 9246-9258. doi: 10.1016/j.biomaterials.2012.09.027

Kalluri, R., and LeBleu, V. S. (2020). The biology, function, and biomedical applications of exosomes. Science 367:eaau6977. doi: 10.1126/science.aau6977

Kassam, A., Bremner, G., Clark, B., Ulibarri, G., and Lennox, R. B. (2006). Place exchange reactions of alkyl thiols on gold nanoparticles. J. Am. Chem. Soc. 128, 3476-3477. doi: 10.1021/ja057091q

Katari, J. E. B., Colvin, V. L., and Alivisatos, A. P. (1994). X-Ray photoelectronspectroscopy Of Cdse nanocrystals with applications to studies of the nanocrystal surface. J. Phys. Chem. 98, 4109-4117. doi: 10.1021/j100066a034

Kobayashi, H., Ogawa, M., Alford, R., Choyke, P. L., and Urano, Y. (2010). New strategies for fluorescent probe design in medical diagnostic imaging. Chem. Rev. 110, 2620-2640. doi: 10.1021/cr900263j

Kojima, R., Bojar, D., Rizzi, G., Hamri, G. C. E., El-Baba, M. D., Saxena, P., et al. (2018). Designer exosomes produced by implanted cells intracerebrally deliver therapeutic cargo for Parkinson's disease treatment. Nat. Commun. 9:1305. doi: 10.1038/s41467-018-03733-8

Krpetic, Z., Nativo, P., Porta, F., and Brust, M. (2009). A multidentate peptide for stabilization and facile bioconjugation of gold nanoparticles. Bioconjug. Chem. 20, 619-624. doi: 10.1021/bc8003028

Kuna, J. J., Voitchovsky, K., Singh, C., Jiang, H., Mwenifumbo, S., Ghorai, P. K., et al. (2009). The effect of nanometre-scale structure on interfacial energy. Nat. Mater. 8, 837-842. doi: 10.1038/nmat2534

Levy, R., Thanh, N. T. K., Doty, R. C., Hussain, I., Nichols, R. J., Schiffrin, D. J., et al. (2004). Rational and combinatorial design of peptide capping Ligands for gold nanoparticles. J. Am. Chem. Soc. 126, 10076-10084. doi: 10.1021/ja0487269

Li, H., Wang, P., Deng, Y. X., Zeng, M. Y., Tang, Y., Zhu, W. H., et al. (2017). Combination of active targeting, enzyme-triggered release and fluorescent dye into gold nanoclusters for endomicroscopy-guided photothermal/photodynamic therapy to pancreatic ductal adenocarcinoma. Biomaterials 139, 30-38. doi: 10.1016/j.biomaterials.2017.05.030

Li, X., Guo, H., Yang, Y., Meng, J., Liu, J., Wang, C., et al. (2014). A designed peptide targeting CXCR4 displays anti-acute myelocytic leukemia activity in vitro and in vivo. Sci. Rep. 4:6610. doi: 10.1038/srep06610

Li, Z. H., Zhao, R. J., Wu, X. H., Sun, Y., Yao, M., Li, J. J., et al. (2005). Identification and characterization of a novel peptide ligand of epidermal growth factor receptor for targeted delivery of therapeutics. Faseb J. 19, 1978-1985. doi: 10. 1096/fj.05-4058com

Libralato, G., Galdiero, E., Falanga, A., Carotenuto, R., de Alteriis, E., and Guida, M. (2017). Toxicity effects of functionalized quantum dots, gold and polystyrene nanoparticles on target aquatic biological models: a review. Molecules 22:1439. doi: 10.3390/molecules22091439

Liu, Z. D., Zhou, C., Qin, Y., Wang, Z. H., Wang, L. Y., Wei, X. L., et al. (2017). Coordinating antigen cytosolic delivery and danger signaling to program potent cross-priming by micelle-based nanovaccine. Cell Discov. 3:17007. doi: 10.1038/ celldisc. 2017.7

Liu, Z., Xiong, M., Gong, J. B., Zhang, Y., Bai, N., Luo, Y. P., et al. (2014). Legumain protease-activated TAT-liposome cargo for targeting tumours and their microenvironment. Nat. Commun. 5:4280. doi: 10.1038/ncomms5280

Long, J. A., Rankin, B. M., and Ben-Amotz, D. (2015). Micelle structure and hydrophobic hydration. J. Am. Chem. Soc. 137, 10809-10815. doi: 10.1021/jacs. 5 b06655

Love, J. C., Estroff, L. A., Kriebel, J. K., Nuzzo, R. G., and Whitesides, G. M. (2005). Self-assembled monolayers of thiolates on metals as a form of nanotechnology. Chem. Rev. 105, 1103-1169. doi: 10.1021/cr0300789

Lu, Y. J., Chuang, E. Y., Cheng, Y. H., Anilkumar, T. S., Chen, H. A., and Chen, J. P. (2019). Thermosensitive magnetic liposomes for alternating magnetic fieldinducible drug delivery in dual targeted brain tumor chemotherapy. Chem. Eng. J. 373, 720-733. doi: 10.1016/j.cej.2019.05.055

Lundquist, A., Wessman, P., Rennie, A. R., and Edwards, K. (2008). Melittin-Lipid interaction: A comparative study using liposomes, micelles and bilayer disks. Biochim. Biophys. Acta Biomembr. 1778, 2210-2216. doi: 10.1016/j.bbamem. 2008.05.009

Ma, W., Cheetham, A. G., and Cui, H. G. (2016). Building nanostructures with drugs. Nano Today 11, 13-30. doi: 10.1016/j.nantod.2015.11.003

Maeda, H., Nakamura, H., and Fang, J. (2013). The EPR effect for macromolecular drug delivery to solid tumors: Improvement of tumor uptake, lowering of 
systemic toxicity, and distinct tumor imaging in vivo. Adv. Drug Deliv. Rev. 65, 71-79. doi: 10.1016/j.addr.2012.10.002

Manna, L., Milliron, D. J., Meisel, A., Scher, E. C., and Alivisatos, A. P. (2003). Controlled growth of tetrapod-branched inorganic nanocrystals. Nat. Mater. 2, 382-385. doi: 10.1038/nmat902

Mao, J. N., Ran, D. N., Xie, C., Shen, Q., Wang, S. L., and Lu, W. Y. (2017). EGFR/EGFRvIII dual-targeting peptide-mediated drug delivery for enhanced glioma therapy. ACS Appl. Mater. Interfaces 9, 24462-24475. doi: 10.1021/ acsami.7b05617

Matsumura, Y., and Maeda, H. (1986). A new concept for macromolecular therapeutics in cancer-chemotherapy - mechanism of tumoritropic accumulation of proteins and the antitumor agent smancs. Cancer Res. 46, 6387-6392.

McNamara, K., and Tofail, S. A. M. (2017). Nanoparticles in biomedical applications. Adv. Phys. X 2, 54-88. doi: 10.1080/23746149.2016.1254570

Meyers, J. D., Cheng, Y., Broome, A. M., Agnes, R. S., Schluchter, M. D., Margevicius, S., et al. (2015). Peptide-targeted gold nanoparticles for photodynamic therapy of brain cancer. Part. Part. Syst. Charact. 32, 448-457. doi: 10.1002/ppsc.201400119

Mittelbrunn, M., Gutierrez-Vazquez, C., Villarroya-Beltri, C., Gonzalez, S., Sanchez-Cabo, F., Gonzalez, M. A., et al. (2011). Unidirectional transfer of microRNA-loaded exosomes from $\mathrm{T}$ cells to antigen-presenting cells. Nat. Commun. 2:282. doi: 10.1038/ncomms 1285

Monopoli, M. P., Aberg, C., Salvati, A., and Dawson, K. A. (2012). Biomolecular coronas provide the biological identity of nanosized materials. Nat. Nanotechnol. 7, 779-786. doi: 10.1038/nnano.2012.207

Moore, T. L., Rodriguez-Lorenzo, L., Hirsch, V., Balog, S., Urban, D., Jud, C., et al. (2015). Nanoparticle colloidal stability in cell culture media and impact on cellular interactions. Chem. Soc. Rev. 44, 6287-6305. doi: 10.1039/c4cs00 $487 \mathrm{f}$

Morshed, R. A., Muroski, M. E., Dai, Q., Wegscheid, M. L., Auffinger, B., Yu, D., et al. (2016). Cell-penetrating peptide-modified gold nanoparticles for the delivery of doxorubicin to brain metastatic breast cancer. Mol. Pharm. 13, 1843-1854. doi: 10.1021/acs.molpharmaceut.6b00004

Nakano, S., Miyoshi, D., and Sugimoto, N. (2014). Effects of molecular crowding on the structures, interactions, and functions of nucleic acids. Chem. Rev. 114, 2733-2758. doi: 10.1021/cr400113m

Noble, G. T., Stefanick, J. F., Ashley, J. D., Kiziltepe, T., and Bilgicer, B. (2014). Ligand-targeted liposome design: challenges and fundamental considerations. Trends Biotechnol. 32, 32-45. doi: 10.1016/j.tibtech.2013.09.007

Owens, D. E., and Peppas, N. A. (2006). Opsonization, biodistribution, and pharmacokinetics of polymeric nanoparticles. Int. J. Pharm. 307, 93-102. doi: 10.1016/j.ijpharm.2005.10.010

Pakulska, M. M., Miersch, S., and Shoichet, M. S. (2016). Designer protein delivery: From natural to engineered affinity-controlled release systems. Science 351:aac4750. doi: 10.1126/science.aac4750

Pal, K., Al-suraih, F., Gonzalez-Rodriguez, R., Dutta, S. K., Wang, E., Kwak, H. S., et al. (2017). Multifaceted peptide assisted one-pot synthesis of gold nanoparticles for plectin-1 targeted gemcitabine delivery in pancreatic cancer. Nanoscale 9, 15622-15634. doi: 10.1039/c7nr03172f

Patra, J. K., Das, G., Fraceto, L. F., Campos, E. V. R., Rodriguez-Torres, M. D. P., Acosta-Torres, L. S., et al. (2018). Nano based drug delivery systems: recent developments and future prospects. J. Nanobiotechnol. 16:71. doi: 10.1186/ s12951-018-0392-8

Peng, Q., Zhang, S., Yang, Q., Zhang, T., Wei, X. Q., Jiang, L., et al. (2013). Preformed albumin corona, a protective coating for nanoparticles based drug delivery system. Biomaterials 34, 8521-8530. doi: 10.1016/j.biomaterials.2013. 07.102

Pitt, J. M., Andre, F., Amigorena, S., Soria, J. C., Eggermont, A., Kroemer, G., et al. (2016). Dendritic cell-derived exosomes for cancer therapy. J. Clin. Invest. 126, 1224-1232. doi: 10.1172/jci81137

Prasuhn, D. E., Blanco-Canosa, J. B., Vora, G. J., Delehanty, J. B., Susumu, K., Mei, B. C., et al. (2010). Combining chemoselective ligation with polyhistidinedriven self-assembly for the modular display of biomolecules on quantum dots. ACS Nano 4, 267-278. doi: 10.1021/nn901393v

Qin, Y., Zhang, Q. Y., Chen, H. L., Yuan, W. M., Kuai, R., Xie, F. L., et al. (2012). Comparison of four different peptides to enhance accumulation of liposomes into the brain. J. Drug Target. 20, 235-245. doi: 10.3109/1061186x.2011.639022
Ramalingam, V. (2019). Multifunctionality of gold nanoparticles: plausible and convincing properties. Adv. Colloid Interface Sci. 271:101989. doi: 10.1016/j.cis. 2019.101989

Reff, M. E., Hariharan, K., and Braslawsky, G. (2002). Future of monoclonal antibodies in the treatment of hematologic malignancies. Cancer Contr. 9, 152-166. doi: 10.1177/107327480200900207

Roy, E., Patra, S., Madhuri, R., and Sharma, P. K. (2017). Carbon dot/TAT peptide co-conjugated bubble nanoliposome for multicolor cell imaging, nucleartargeted delivery, and chemo/photothermal synergistic therapy. Chem. Eng. J. 312, 144-157. doi: 10.1016/j.cej.2016.11.122

Salvati, A., Pitek, A. S., Monopoli, M. P., Prapainop, K., Bombelli, F. B., Hristov, D. R., et al. (2013). Transferrin-functionalized nanoparticles lose their targeting capabilities when a biomolecule corona adsorbs on the surface. Nat. Nanotechnol. 8, 137-143. doi: 10.1038/nnano.2012.237

Sapsford, K. E., Algar, W. R., Berti, L., Gemmill, K. B., Casey, B. J., Oh, E., et al. (2013). Functionalizing nanoparticles with biological molecules: developing chemistries that facilitate nanotechnology. Chem. Rev. 113, 1904-2074. doi: $10.1021 / \mathrm{cr} 300143 \mathrm{v}$

Sasikumar, P. G., and Ramachandra, M. (2018). Small-molecule immune checkpoint inhibitors targeting PD-1/PD-L1 and other emerging checkpoint pathways. Biodrugs 32, 481-497. doi: 10.1007/s40259-018-0303-4

Sauer, I., Nikolenko, H., Keller, S., Abu Ajaj, K., Blenert, M., and Dathe, M. (2006). Dipalmitoylation of a cellular uptake-mediating apolipoprotein E-derived peptide as a promising modification for stable anchorage in liposomal drug carriers. Biochim. Biophys. Acta Biomembr. 1758, 552-561. doi: 10.1016/j. bbamem.2006.03.017

Schwarze, S. R., Ho, A., Vocero-Akbani, A., and Dowdy, S. F. (1999). In vivo protein transduction: delivery of a biologically active protein into the mouse. Science 285, 1569-1572. doi: 10.1126/science.285.5433.1569

Singh, C., Ghorai, P. K., Horsch, M. A., Jackson, A. M., Larson, R. G., Stellacci, F., et al. (2007). Entropy-mediated patterning of surfactant-coated nanoparticles and surfaces. Phys. Rev. Lett. 99:226106. doi: 10.1103/PhysRevLett.99.226106

Singh, C., Hu, Y., Khanal, B. P., Zubarev, E. R., Stellacci, F., and Glotzer, S. C. (2011). Striped nanowires and nanorods from mixed SAMS. Nanoscale 3, 3244-3250. doi: 10.1039/c1nr10215j

Song, X., Wan, Z. Y., Chen, T. J., Fu, Y., Jiang, K. J., Yi, X. L., et al. (2016). Development of a multi-target peptide for potentiating chemotherapy by modulating tumor microenvironment. Biomaterials 108, 44-56. doi: 10.1016/ j.biomaterials.2016.09.001

Spicer, C. D., Jumeaux, C., Gupta, B., and Stevens, M. M. (2018). Peptide and protein nanoparticle conjugates: versatile platforms for biomedical applications. Chem. Soc. Rev. 47, 3574-3620. doi: 10.1039/c7cs00877e

Sugahara, K. N., Teesalu, T., Karmali, P. P., Kotamraju, V. R., Agemy, L., Girard, O. M., et al. (2009). Tissue-penetrating delivery of compounds and nanoparticles into tumors. Cancer Cell 16, 510-520. doi: 10.1016/j.ccr.2009.10. 013

Teesalu, T., Sugahara, K. N., Kotamraju, V. R., and Ruoslahti, E. (2009). C-end rule peptides mediate neuropilin-1-dependent cell, vascular, and tissue penetration. Proc. Natl. Acad. Sci. U.S.A. 106, 16157-16162. doi: 10.1073/pnas.0908201106

Terrill, R. H., Postlethwaite, T. A., Chen, C. H., Poon, C. D., Terzis, A., Chen, A. D., et al. (1995). Monolayers in three dimensions: NMR, SAXS, thermal, and electron hopping studies of alkanethiol stabilized gold clusters. J. Am. Chem. Soc. 117, 12537-12548. doi: 10.1021/ja00155a017

Thevenin, D., An, M., and Engelman, D. M. (2009). pHLIP-mediated translocation of membrane-impermeable molecules into cells. Chem. Biol. 16, 754-762. doi: 10.1016/j.chembiol.2009.06.006

Thorpe, P. E. (2004). Vascular targeting agents as cancer therapeutics. Clin. Cancer Res. 10, 415-427. doi: 10.1158/1078-0432.ccr-0642-03

Tung, C., and Cacciuto, A. (2013). Phase separation of mixed polymer brushes on surfaces with nonuniform curvature. J. Chem. Phys. 139:194902. doi: 10.1063/1. 4829638

Valadi, H., Ekstrom, K., Bossios, A., Sjostrand, M., Lee, J. J., and Lotvall, J. O. (2007). Exosome-mediated transfer of mRNAs and microRNAs is a novel mechanism of genetic exchange between cells. Nat. Cell Biol. 9, 654-672. doi: $10.1038 /$ ncb1596

Verdurmen, W. P. R., Mazlami, M., and Pluckthun, A. (2017). A quantitative comparison of cytosolic delivery via different protein uptake systems. Sci. Rep. 7:13194. doi: 10.1038/s41598-017-13469-y 
Vlieghe, P., Lisowski, V., Martinez, J., and Khrestchatisky, M. (2010). Synthetic therapeutic peptides: science and market. Drug Discov. Today 15, 40-56. doi: 10.1016/j.drudis.2009.10.009

Vo, T. N., Kasper, F. K., and Mikos, A. G. (2012). Strategies for controlled delivery of growth factors and cells for bone regeneration. Adv. Drug Deliv. Rev. 64, 1292-1309. doi: 10.1016/j.addr.2012.01.016

Walkey, C. D., and Chan, W. C. W. (2012). Understanding and controlling the interaction of nanomaterials with proteins in a physiological environment. Chem. Soc. Rev. 41, 2780-2799. doi: 10.1039/c1cs15233e

Wang, C. X., Biok, N. A., Nayani, K., Wang, X. G., Yeon, H., Ma, C. K. D., et al. (2021). Cationic side chain identity directs the hydrophobically driven self-assembly of amphiphilic beta-peptides in aqueous solution. Langmuir 37, 3288-3298. doi: 10.1021/acs.langmuir.0c03255

Wang, P., Zhang, L. M., Zheng, W. F., Cong, L. M., Guo, Z. R., Xie, Y. Z. Y., et al. (2018). Thermo-triggered release of CRISPR-Cas9 system by lipid-encapsulated gold nanoparticles for tumor therapy. Angew. Chem. Int. Ed. 57, 1491-1496. doi: 10.1002/anie.201708689

Webb, M. S., Saxon, D., Wong, F. M. P., Lim, H. J., Wang, Z., Bally, M. B., et al. (1998). Comparison of different hydrophobic anchors conjugated to poly(ethylene glycol): effects on the pharmacokinetics of liposomal vincristine. Biochim. Biophys. Acta Biomembr. 1372, 272-282. doi: 10.1016/s0005-2736(98) 00077-7

Wilhelm, S., Tavares, A. J., Dai, Q., Ohta, S., and Chan, W. C. W. (2016). Analysis of nanoparticle delivery to tumours. Nat. Rev. Mater. 1:16014. doi: 10.1038/ natrevmats.2016.14

Witzigmann, D., Uhl, P., Sieber, S., Kaufman, C., and Huwyler, J. (2019). Optimization-by-design of hepatotropic lipid nanoparticles targeting the sodium-taurocholate cotransporting polypeptide. eLife Ences 8:e42276. doi: 10. 7554/eLife.42276

Wu, R. H., Nguyen, T. P., Marquart, G. W., Miesen, T. J., Mau, T., and Mackiewicz, M. R. (2014). A facile route to tailoring peptide-stabilized gold nanoparticles using glutathione as a synthon. Molecules 19, 6754-6775. doi: 10.3390/molecules19056754

Wu, Y., Ali, M. R. K., Chen, K. C., Fang, N., and El-Sayed, M. A. (2019). Gold nanoparticles in biological optical imaging. Nano Today 24, 120-140. doi: 10. 1016/j.nantod.2018.12.006

Xie, J., Bi, Y., Zhang, H., Dong, S. Y., Teng, L. H., Lee, R. B. J., et al. (2020). Cell-penetrating peptides in diagnosis and treatment of human diseases: from preclinical research to clinical application. Front. Pharmacol. 11:697. doi: 10. 3389/fphar.2020.00697

Xie, C., Upputuri, P. K., Zhen, X., Pramanik, M., and Pu, K. Y. (2017). Self-quenched semiconducting polymer nanoparticles for amplified in vivo photoacoustic imaging. Biomaterials 119, 1-8. doi: 10.1016/j.biomaterials.2016. 12.004

Xu, J., Ruchala, P., Ebenstain, Y., Li, J. J., and Weiss, S. (2012). Stable, compact, bright biofunctional quantum dots with improved peptide coating. J. Phys. Chem. B 116, 11370-11378. doi: 10.1021/jp306453y
Yan, H., Zhong, G. C., Xu, G. W., He, W. H., Jing, Z. Y., Gao, Z. C., et al. (2012). Sodium taurocholate cotransporting polypeptide is a functional receptor for human hepatitis B and D virus. Elife 1:28. doi: 10.7554/eLife.00049

Yang, L. J., Zhang, C. R., Ren, C. H., Liu, J. J., Zhang, Y. M., Wang, J., et al. (2019). Supramolecular hydrogel based on chlorambucil and peptide drug for cancer combination therapy. ACS Appl. Mater. Interfaces 11, 331-339. doi: 10.1021/acsami.8b18425

Yang, L. Q., Li, M. M., Sun, Y. S., and Zhang, L. F. (2018). A cellpenetrating peptide conjugated carboxymethyl-beta-cyclodextrin to improve intestinal absorption of insulin. Int. J. Biol. Macromol. 111, 685-695. doi: 10.1016/j.ijbiomac.2018.01.077

Yu, L. L., Zhang, W. B., Luo, W. D., Dupont, R. L., Xu, Y., Wang, Y. B., et al. (2020). Molecular recognition of human islet amyloid polypeptide assembly by selective oligomerization of thioflavin T. Sci. Adv. 6:8. doi: 10.1126/sciadv.abc1449

Yu, M. K., Park, J., and Jon, S. (2012). Targeting strategies for multifunctional nanoparticles in cancer imaging and therapy. Theranostics 2, 3-44. doi: 10.7150/ thno.3463

Zhang, X. X., Eden, H. S., and Chen, X. (2012). Peptides in cancer nanomedicine: drug carriers, targeting ligands and protease substrates. J. Control Release 159, 2-13. doi: 10.1016/j.jconrel.2011.10.023

Zhao, T., Zhou, H., Lei, L., Guo, C., Yang, Q., Gong, T., et al. (2020). A new tandem peptide modified liposomal doxorubicin for tumor "ecological therapy". Nanoscale 12, 3359-3369. doi: $10.1039 /$ c9nr09585c

Zheng, Y., Fang, X., Yang, Y., and Wang, C. (2021). Peptide-directed delivery of drug-loaded nanocarriers targeting CD36 overexpressing cells. Colloids Surf. A Physicochem. Eng. Aspects 610, 125970. doi: 10.1016/j.colsurfa.2020.125970

Zou, L. L., Ding, W. P., Zhang, Y. Y., Cheng, S. H., Li, F. F., Ruan, R. Q., et al. (2018). Peptide-modified vemurafenib-loaded liposomes for targeted inhibition of melanoma via the skin. Biomaterials 182, 1-12. doi: 10.1016/j.biomaterials. 2018.08.013

Zu, R. J., Fang, X. C., Lin, Y. C., Xu, S. L., Meng, J., Xu, H. Y., et al. (2020). Peptide-enabled receptor-binding-quantum dots for enhanced detection and migration inhibition of cancer cells. J. Biomater. Sci. Polymer Ed. 31, 1604-1621. doi: 10.1080/09205063.2020.1764191

Conflict of Interest: The authors declare that the research was conducted in the absence of any commercial or financial relationships that could be construed as a potential conflict of interest.

Copyright (c) 2021 Liu, Fang, Yang and Wang. This is an open-access article distributed under the terms of the Creative Commons Attribution License (CC BY). The use, distribution or reproduction in other forums is permitted, provided the original author(s) and the copyright owner(s) are credited and that the original publication in this journal is cited, in accordance with accepted academic practice. No use, distribution or reproduction is permitted which does not comply with these terms. 